\title{
Algebraic combinatorics in bounded induction
}

\section{Joaquín Borrego-Díaz}

Departamento de Ciencias de la Computación e Inteligencia Artificial, E.T.S. Ingeniería Informática, Universidad de Sevilla, Avda. Reina Mercedes s.n., 41012, Sevilla, Spain

\author{
A B S T R A C T
}

Keywords:

Bounded induction

Stone-Čech compactification

Ramsey theorem

Peano Arithmetic
In this paper, new methods for analyzing models of weak subsystems of Peano Arithmetic are proposed. The focus will be on the study of algebro-combinatoric properties of certain definable cuts. Their relationship with segments that satisfy more induction, with those limited by the standard powers/roots of an element, and also with definable sets in Bounded Induction is studied. As a consequence, some considerations on the $\Pi_{1}$-interpretability of $\mathrm{I} \Delta_{0}$ in weak theories, as well as some alternative axiomatizations, are reviewed. Some of the results of the paper are obtained by immersing Bounded Induction models in its Stone-Cech Compactification, once it is endowed with a topology.

\section{Contents}

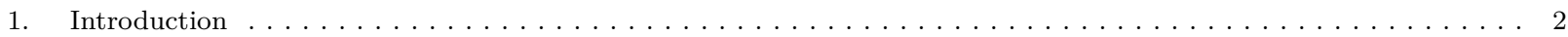

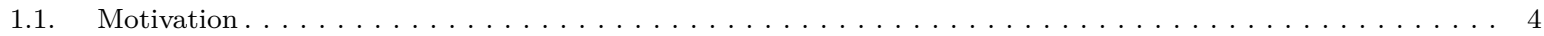

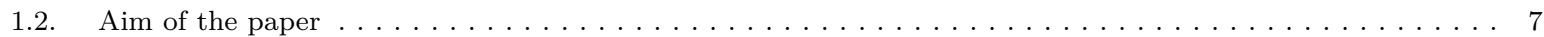

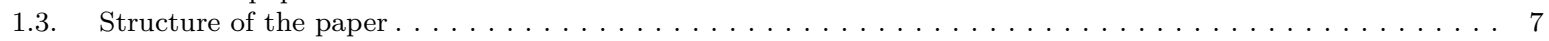

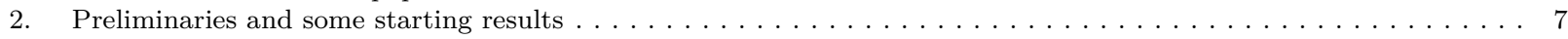

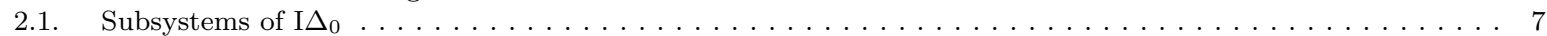

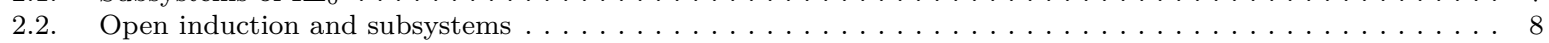

2.3. Exponentiation and segments determined by (standard) powers or integer roots . . . . . . . . . . . 9

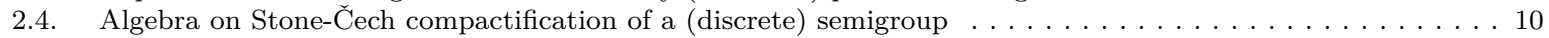

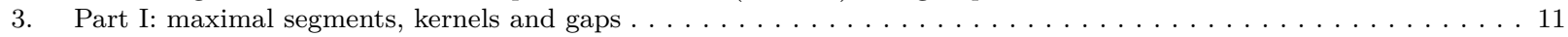

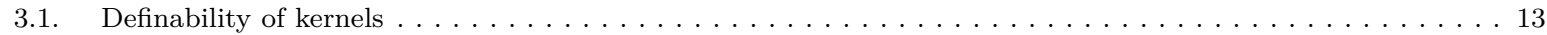

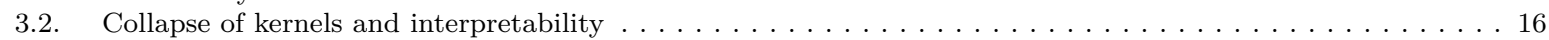

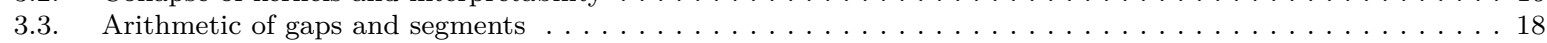

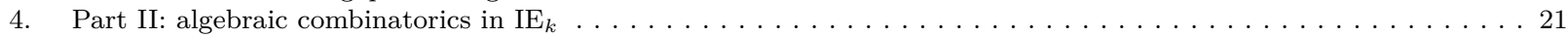

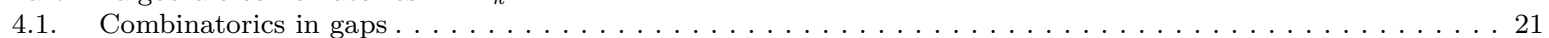




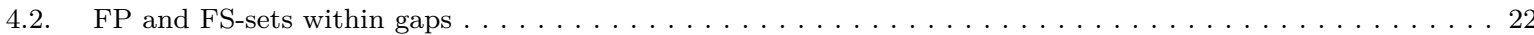

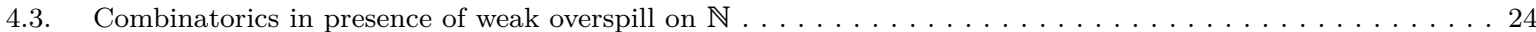

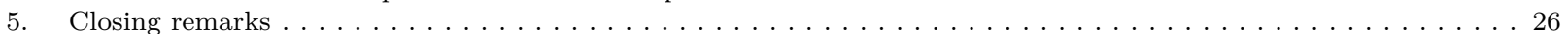

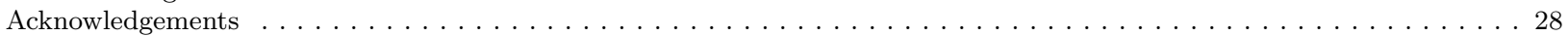

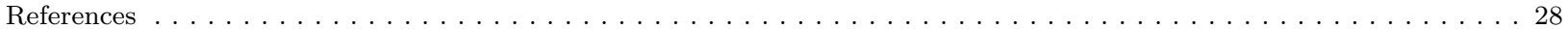

\section{Introduction}

A significant amount of the research corpus on (models of) classic subsystems of Peano Arithmetic (PA) is primarily focused on achieving two types of goals. A first type refers to studying how powerful a theory (e.g. fragment of PA) is for supporting the results of elementary number theory. A second one is devoted to obtaining independence results for (open) central problems to Complexity Theory [16]. The first objective is usually focused on theories weaker than $\mathrm{I} \Sigma_{1}$, because that theory is already enough to develop a large part of Number Theory. A frontier for such an analysis is Parikh's theory I $\Delta_{0}$ (the theory of bounded induction), where several problems are related to its power for developing number theory remains open, some of these being related to open problems in Complexity Theory. Its "frontier" nature is because a large number of these questions can be solved if $\mathrm{I} \Delta_{0}$ is extended with an axiom that ensures that a certain total function grows faster than any polynomial function. For example, the exponential function. Please let us recall that according to a celebrated result of R. Parikh this kind of functions are not definable in I $\Delta_{0}$ (cf. [22], Th. V.1.4):

Result 1.1. Every provably recursive function in $\mathrm{I} \Delta_{0}$ is bounded by a polynomial.

Concerning the second research line (the searching for independence results), the situation is similar. For example, in strong fragments, a classical approach to solve this type of problems is based on showing the existence, in any model of the subsystem, of an initial segment (or final extension), that is a model of a stronger theory. This technique is useful to separate theories [40], as well as for connecting model-theoretic and complexity issues (see e.g. the survey [38]). In the same way as in the first research topic, when one works with fragments of PA, almost all positive results are also limited to proper extensions of I $\Delta_{0}$ (such as $\mathrm{I} \Sigma_{n+1}$ or $\left.\mathrm{B} \Sigma_{n+1}\right)$.

The scenario substantially changes when the theories $\left\{\mathrm{IE}_{n}\right\}_{n \in \omega}$ (fragments of $\mathrm{I}_{0}$ defined like $\mathrm{I} \Sigma_{n}$ but using bounded quantifiers instead) are considered. It is not known if this hierarchy is strict. In fact there are relatively very few results about the relationship between $\mathrm{IE}_{k}$ and $\mathrm{IE}_{k+1}(k \geq 1)$. In stronger subsystems, global coding tools are used to prove results, but this type of functions are not definable in Bounded Induction by Result 1.1, so they can not be used (in particular, arithmetization).

We could affirm that one of the reasons for this situation is that the relationship of these theories with classes of computational complexity is not so evident to be able to translate problems between both fields. Let us consider, for example, the case of the class of provably recursive theories on $\mathrm{IE}_{n}$. In general, a useful approach to studying fragments of PA is to consider, given a $T$ theory, the class $R(T)$ of provably recursive functions in $T$ and to study, for example, the existence of initial segments that are closed under that class of functions in (some) Arithmetic models (see [9] for a refinement of up to $\mathrm{IE}_{1}$ ). However, the approach could be not useful here in our framework, because $\left\{R\left(\mathrm{IE}_{n}\right)\right\}_{n<\omega}$ collapses.

Proposition 1.2. $R\left(\mathrm{I} \Delta_{0}\right)=R\left(\mathrm{PA}^{-}\right)$.

Proof. (taken from [4]) Assume that $f$ is provably recursive in $\mathrm{IE}_{n}$ by the formula $\exists z \theta(x, y, z)$, with $\theta \in \mathrm{E}_{k}$. Then there is a $\Pi_{1}$-sentence $\forall u \psi(u)$ (suppose $\psi \in \mathrm{E}_{n+1}$ ), such that $\mathrm{PA}^{-}+\forall u \psi(u) \vdash \forall x \exists ! y \exists z \theta(x, y, z)$. Then

$$
\mathrm{PA}^{-} \vdash \forall u \psi(u) \rightarrow \forall x \exists v \exists y, z<v \varphi(x, y, z, v)
$$


where $\varphi \in \mathrm{E}_{k+1}$ is

$$
\varphi(x, y, z, v):=\theta(x, y, z) \wedge \forall y_{1}, y_{2}, z_{1}, z_{2}<v\left(\theta\left(x, y_{1}, z_{1}\right) \wedge \theta\left(x, y_{2}, z_{2}\right) \rightarrow y_{1}=y_{2}\right)
$$

Therefore,

$$
\mathrm{PA}^{-} \vdash \forall x \exists v \exists u(\neg \psi(u) \vee \exists y, z<v \varphi(x, y, z, v))
$$

By 1.1, there is $p(x) \in \mathbb{N}[x]$ that bounds $v, u$. Hence the $\mathrm{E} \max \{n+3, k+1\}-$ formula

$$
\gamma(x, y, z) \equiv \exists v<p(x)\left\{\begin{array}{l}
\forall u \leq p(x) \psi(u) \wedge \varphi(x, y, z, v) \wedge y<v \wedge z<v \\
\vee \\
\exists u \leq p(x) \neg \psi(u) \wedge y=0
\end{array}\right.
$$

represents the standard graph of $f$ and $\mathrm{PA}^{-} \vdash \forall x \exists ! y \exists z \gamma(x, y, z)$.

A plausible way to attack the problem of the possible collapse of $\left\{\mathrm{IE}_{n}\right\}_{n \in \omega}$ (or, for instance, whether $\left.\mathrm{IE}_{1} \vdash \mathrm{IE}_{2}\right)$ is to find intermediate alternative axiom schemes to facilitate the analysis. Due to the essentially different behavior of the recursive function classes in Bounded Induction, one option might be to design schemes that describe algebraic properties or properties about, for example, the order $<$ over $\mathbb{N}$. This idea has been used frequently in the strong fragments. In [36] Kreuzer and Yokoyama show interesting intermediate schemes between $I \Sigma_{1}$ and $I \Sigma_{2}$ of different nature, which are equivalent if $I \Sigma_{1}$ is considered as the base theory, and which are equivalent in turn to the well-foundedness of $\omega^{\omega}$.

Among the published works on $\mathrm{IE}_{n}$, some of them deserve to be highlighted. There are refinements of proven facts for $\mathrm{I} \Sigma_{n}$ (for instance the existence in nonstandard models of $\mathrm{IE}_{1}$ of a nonstandard initial segment model of PA [29,42]), studies of algebraic nature (see e.g. [5,43] for $\mathrm{IE}_{0}$ and [44] for $\mathrm{IE}_{1}$ ) and other studies about alternative axiomatizations $[3,15]$. Whether $\mathrm{I} \Delta_{0}$ is finitely axiomatizable -or whether $\left\{\mathrm{IE}_{n}\right\}_{n<\omega}$ collapses- is the most important open problem in the field, connected with the $P=N P$ ? problem (cf. $[7,22])$.

A noticeable result about the theories $\mathrm{IE}_{n}$ shall be used in this paper (due to Paris and Dimitracopoulos [14], that is proved by using a formalization of truth definition for $\mathrm{E}_{n}$-formulas):

Result 1.3. For all $n$ there is a $\Pi_{1}$-sentence $\sigma_{n}$ such that

$$
\mathrm{I} \Delta_{0} \vdash \sigma_{n} \vdash \mathrm{IE}_{n}
$$

Please, let us note that the Result 1.3 implies that, if $\left\{\mathrm{IE}_{n}\right\}_{n}$ collapses then $\mathrm{I} \Delta_{0}$ is finitely axiomatizable. Result 1.3 has been used by R. Kaye for studying models of $\mathrm{IE}_{1}$ [29].

The above result is useful for our purposes because the reciprocal one -i.e., looking for a property that, added to $\mathrm{IE}_{n}$ a theory equivalent to $\mathrm{I} \Delta_{0}$ can be obtained- is more difficult to study in general, because it is possible that adding to $\mathrm{IE}_{1}$ a numerical property (or for example Ramsey type scheme) stronger theories than the I $\Delta_{0}$ itself are obtained. Two examples would be [10] (numerical property) and [8] (Ramsey type scheme). In the first paper, P. D'Aquino described the difference between $\mathrm{IE}_{1}$ and $\mathrm{I}_{0}+\exp$ employing a property of number theory:

$$
\mathrm{IE}_{1}+P \vdash \mathrm{I} \Delta_{0}+\exp
$$

where $P$ is an axiom that states that every Pell equation has a solution. In the second one, C. Cornaros studied the strength of weak forms of the Regularity Principle in the presence of $\mathrm{IE}_{1}$, proving that a Bounded Weak Regularity Principle on $E_{1}$ formulas is equivalent -over $\mathrm{IE}_{1}-$ to $\mathrm{I} \Delta_{0}+$ exp. 
In general, to investigate the relationship between the theorems in $\mathrm{PA}$ (or $\mathrm{I} \Delta_{0}$ ) and those of $\mathrm{PA}^{-}$it is common to focus on the inductive formula(s) necessary to demonstrate the corresponding theorem.

Definition 1.4. A formula $\varphi(x)$ is called inductive if $P A^{-} \vdash \varphi(0)$ and $P A^{-} \vdash \forall x(\varphi(x) \rightarrow \varphi(x+1))$.

The analysis for the inductive formulas is interesting by itself, as well as the study of their behavior in a model [23]. An interesting result for this paper is the following (in Section 3.2 variants of the result using a fixed inductive formula will be studied).

Theorem 1.5. (Wilkie-Paris, see [50]) Let $\varphi(x)$ be a bounded formula. The following conditions are equivalent:

1. $\mathrm{I} \Delta_{0}+\exp \vdash \forall x \varphi(x)$

2. There exists an inductive formula $\psi$ such that $P A^{-} \vdash \forall x(\psi(x) \rightarrow \varphi(x))$

\subsection{Motivation}

The underlying general hypothesis that guides this work is that to advance in the study of (very weak) subsystems of bounded induction it could be interesting to explore the design and use of new tools that exploit properties of natural numbers that are essentially different in nature from those of classical fragments. For example, techniques from other research fields that are not traditionally related to the study of arithmetic models (such as the topology of semigroups), thus changing the point of view with which the models are analyzed.

The working hypothesis is relevant when the interest is on theories that extend very weak theories: $\mathrm{IE}_{0}^{1}$, the extension of $\mathrm{PA}^{-}$allowing Euclidean division; and $\mathrm{RIE}_{0}^{1}$, that extends it assuring the existence of integer roots. Therefore, we will work with the absence of definable functions of rapid growth (that is, asymptotically faster than any polynomial) which obstructs us from using global arithmetization techniques (that are useful to reproduce classical demonstrations).

Concerning the selection of algebraic properties that may be interesting and how to use them, let us consider the paradigmatic case of the set of prime numbers. It is unknown whether $\mathrm{I} \Delta_{0}$ demonstrates its infinitude. Thus, whilst we cannot assume that this set is unbounded in a $\mathrm{I} \Delta_{0}$ model, some combinatorial properties associated with its infinity in the standard model can be studied. For example, a very interesting combinatorial property of such a set is that it contains arbitrarily long arithmetic progressions (a celebrated result of Green and Tao [47], see also [19]). We intend to approach the study of properties of this type using some results of the Ramsey type.

In general terms, Ramsey type theorems state that if the universe to be studied is divided into a series of parts, then at least one of them enjoys a particular property (commonly associated with a certain kind of regularity). In the finite case, Ramsey's theory refers to how large the set of parts must be to ensure that the property is satisfied, while in the infinite case the properties focus on detecting a certain regularity in one of those parts. Unlike the seminal result -Ramsey's theorem- these properties can now be studied with non-standard methods (see e.g. [26]). A well-known example on that line is Schur's theorem: given $n>0$, for any prime number $p$ large enough there is a non-trivial solution of $x^{n}+y^{n}=z^{n}(\bmod p)$. A nice proof is based on the fact that there are three monochromatic elements in a certain $\mathbb{N}$ partition (a Ramsey property). Although there are studies of the peculiarities about Ramsey Theorem on theories weaker than $\mathrm{I} \Sigma_{1}$ (see for example [52]), in general, these are focused on theories stronger than $\mathrm{I} \Delta_{0}$.

We are concerned with two general questions. On the one hand, are these types of combinatorial properties on infinite sets (actually, a suitable version) provable in Bounded Induction, independently of the ignorance about the provability of their infinitude? On the other hand, if it is possible to demonstrate a 


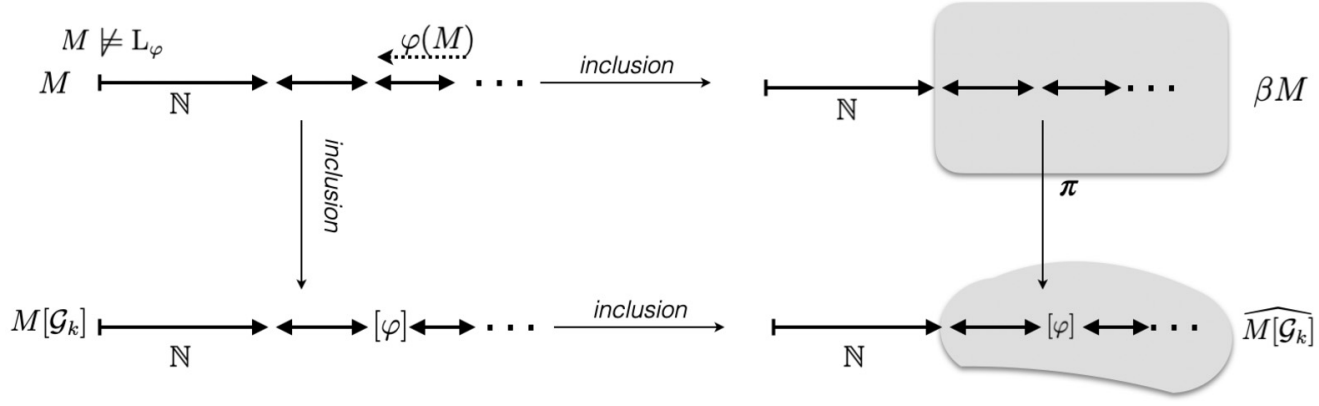

Fig. 1. Compactification of the model augmented with $\Delta_{0}$-definable sets without first element.

result of (algebro-)combinatorial regularity of that type, what is the relationship between its corresponding axiomatization as an axiom scheme and $\mathrm{I} \Delta_{0}$ itself? It should be noted that the results of a combinatorial nature had already been obtained. To quote a recent one, the work of C. Cornaros [8] on the principle of regularity for bounded formulas. The principle of regularity states that if the elements of an infinite set are colored with a finite palette then there is a monochromatic infinite subset. Regularity might be seen as a result of Ramsey type.

To simplify the handling of these types of sets and their properties we will approach the question in a dual (although equivalent) way. Instead of working with combinatorial properties of bounded inductive sets in a model of weak arithmetic, we will work with nonempty sets without the first element instead (i.e., the corresponding minimization principle fails).

Combinatorial properties of that nature (those showing remarkable algebraic regularities in certain infinite sets) can be attacked by working on an extension of the model that enables us to work within a richer structure. A widely used choice in the case of $\mathbb{N}$ is its Stone-Čech compactification, $\beta \mathbb{N}$, which has proved to be an extremely useful structure for rewriting properties of Combinatorial Number Theory in another algebraic (topological) ones about $\beta \mathbb{N}$. Typically, it can be rewritten in terms of the existence of a specific ultrafilter (or a point in another type of compactification). Next, the corresponding property on $\beta \mathbb{N}$ can be addressed using very powerful techniques (see the surveys [2,13,24], or the recent example [45] where the author analyzes of the mentioned set of prime numbers from that perspective). Our interest in $\beta \mathbb{N}$ (or $\beta M$ being $M$ a model of weak arithmetic) lies in the fact that there are strong relationships between the infinite sets in $\mathbb{N}$ and elements (ultrafilters) in $\beta \mathbb{N}$ (as expected for the construction of this one). Infinite sets of $\mathbb{N}$ induce open neighbors in $\beta \mathbb{N}$ that contains non-principal ultrafilters with interesting properties. The selection of a suitable ultrafilter will allow to establish the Ramsey type discussed findings.

Continuing with the idea, a model $M \models P A^{-}$can be viewed as an ordered semigroup, thus it is susceptible to being studied algebraically. If the model is endowed with a topology (for example the discrete one), results and techniques developed for topological semigroups can be used. The potential application would not be interesting if, additionally, if one does not work on an adequate compactification of the structure, in order to obtain a richer topology than the discrete one, with interesting properties as $\beta M$. We think that we have in that way a certain useful analogy between this kind of compactness and saturation in models of Arithmetic. Both properties allow assuring the existence of a certain element if an adequate approximation to it (a sequence in the compact case or a finitely satisfiable type in the case of saturation) can be obtained. We are particularly interested in that relationship for the special case of Stone-Cech compactification $\beta M$.

More precisely, the classic construction of $\beta \mathbb{N}$ has inspired us the following framework (shown in Fig. 1). In more detail, a model of Arithmetic $M$ is extended to a structure $M\left[\mathcal{G}_{k}\right]$ where the $\Delta_{0}$-definable sets without minimum are also elements; they will be interpreted in a similar way to Henkin's cuts. The notion of cut induces a definable equivalence relationship $\sim$ between that sets (two sets are equivalent if they induce the same cut), switching from that mode to working with equivalency classes $[\varphi]$. 
The set $M\left[\mathcal{G}_{k}\right]$ is endowed with the Hausdorff topology generated by the basis:

$$
\{\{a\}\} \text { if } a \in M \text { and }\left\{(a, b) \subseteq M\left[\mathcal{G}_{k}\right]: M \models a<\varphi<b\right\} \text { if }[\varphi] \notin M
$$

(Please, let us note that $M\left[\mathcal{G}_{k}\right]$ under the equivalence relation $\sim$ is the so-called Kolmogorov quotient of this topology.)

With that topology the set $M$ is dense in $M\left[\mathcal{G}_{k}\right]$. Since $\beta M$ is the biggest compactification of $M$, there is a projection

$$
\pi: \beta M \rightarrow \widehat{M\left[\mathcal{G}_{k}\right]}
$$

between $\beta M$ and any right-topological semigroup compactification of $M\left[\mathcal{G}_{k}\right]$ we select (cf. th. 21.4 of [24]). Since $\pi \uparrow_{M}=I d_{M}$ is an homomorphism between dense sets ( $\beta M$ is endowed with the semigroup structure that it will be described in Sect. 2.4), then $\pi$ is also homomorphism (cf. th. 4.22 of [24]).

The construction is interesting basically because the sets without the first element become elements of the structure, thus we are going to be able to draw information from those sets by studying (subsets of) the semigroup $\mathbb{S}_{\varphi}:=\pi^{-1}(\{[\varphi]\})$, when $[\varphi]$ is an idempotent element in $M\left[\mathcal{G}_{k}\right]$. Then some well-known results about $\beta M$ as compact right topological semigroup can be applied. Although the reference to $\left.\widehat{M\left[\mathcal{G}_{k}\right.}\right]$ can be omitted throughout the paper by defining the subsemigroups only in terms of $\beta M$ (as we do in fact below), we believe that the semigroup $\mathbb{S}_{\varphi}$ has interest itself, as it will be commented in the closing remarks of this paper.

Specifically, we are interested in translating a basic property of $\mathbb{N}$ related to the existence of relative primes in a set (i.e., elements of the set that can not be decomposed as a product of numbers from the set itself). Given $A \neq \emptyset$, let $S(A)$ be the set of its lower bounds,

$$
S(A)=\{x: \forall b \in A(x<b)\}
$$

Given two sets $A, B$, it will be said that they define the same cut, $A \sim_{S} B$, if $S(A)=S(B)$. Let $A^{*}$ be the set of its relative composite elements,

$$
A^{*}:=(A \cdot A) \cap A=\{x \in A: \exists b, c \in A(x=b \cdot c)\}
$$

A basic property of $\mathbb{N}$ is that if $0,1 \notin A$, then $A^{*} \nsim_{S} A$. It is not hard to prove that I $\Delta_{0}$ is enough to prove that fact for any $\Delta_{0}$-definable set. However, the reciprocal (namely if that property characterizes $\mathrm{I} \Delta_{0}$ ), is somewhat more complicated. We must justify that we can limit ourselves to validate the principle of minimization for sets with adequate internal arithmetic structure to be able to apply that property. In general terms, in this paper alternative axiomatizations of bounded induction theories are obtained through schemes that describe the following property, for any non-empty $\Delta_{0}$-definable set:

$$
A \sim_{S} A^{*} \Rightarrow\{0,1\} \cap A \neq \emptyset
$$

The necessary combinatorial properties will be demonstrated by analyzing some appropriate ultrafilters of the set $\mathbb{S}_{\varphi}$ described above.

If we focus on the idea of exploiting the algebraic properties of the compactification $\beta M$, it is important to note that the behavior of the operations (which are extensions that of $M$ ) is very different from the original ones. For example, in [25] Hindman, Maleki and Strauss shown that if $a$ and $b$ be distinct positive integers, then the equation $u+a \cdot p=v+b \cdot p$ has no trivial solutions with $u, v \in \beta \mathbb{N}$ and $p \in \beta \mathbb{N} \backslash \mathbb{N}$ (in that paper the abelian groups where the corresponding equation holds in their corresponding Stone-Čech compactifications are characterized). However, other interesting relationships have been proven (see e.g. the 
survey [24]). More details of this issue -once formalized- will be shown in the subsection 2.4. On the other hand, other results do establish a certain transfer of properties (for example that of [39]), although in general, it is not obtained straightforwardly. For example, for any sufficiently strong theory of arithmetic, the set of Diophantine equations provably unsolvable in the theory is algorithmically undecidable, as a consequence of the MRDP theorem. In contrast, in [27] the author proves the decidability of Diophantine equations provably unsolvable in Robinson's arithmetic Q. In that paper, the author axiomatizes the universal fragment of $\mathbb{Q}$ itself.

\subsection{Aim of the paper}

Driven by the above motivations, some methods for analyzing fragments of Bounded Induction, which are not refinements of those used for classic fragments (those that were applied to $\mathrm{I} \Sigma_{n}$ or $\mathrm{B} \Sigma_{n}$ ) are presented. The proposal is developed in two stages. In the first part, several results about $\Delta_{0}$-definable sets will be established. The model will be submerged in a more complex structure in the second part (the aforementioned Stone-Čech compactification), where techniques related to algebraic combinatorics will be applied, taking into account the $\Delta_{0}$-definability of the sets involved in the study.

\subsection{Structure of the paper}

As we discussed, the paper has a differentiated structure in two related parts. The first part is dedicated to the analysis of segments and cuts in weak induction models, to provide these sets with an algebraic structure (semi-group). In the second part, the construction will be used to submerge the model in a topological structure (compact topological semigroup) where results of a very powerful combinatorial nature are available. When applied, it will provide information on the structure of the model as well as alternative axiomatizations of weak induction.

Specifically, the content of the paper is as follows. Section 2 is devoted to listing the main elements and results used in the paper. Next, it is studied the so-called T-kernel, the biggest initial segment of a model of a weak theory which is a model of $T$, when $T=\mathrm{IE}_{n}$, studying its approximation by means algebraic initial segments as well as its definability (Sect. 3). Section 4 presents how to show the maximal character of I $\Delta_{0}$ as possible $\Pi_{1}$-interpretable theory in $\operatorname{RIE}_{0}^{1}$ (3.18).

The second part of the paper starts presenting an algebraic (sec. 5) and topological (sec. 4) treatment, including Ramsey theoretic arguments, of the upper neighborhood of a bounded definable set without the first element. This study allows to give alternative axiomatizations of I $\Delta_{0}$ representing the property $(\diamond)$ described above, as well as to state combinatorial regularities near to the kernel.

\section{Preliminaries and some starting results}

In this section, a number of notions and results to be used throughout the paper will be introduced. Specifically, some results on $\mathrm{I}_{0}, \mathrm{IE}_{k}(k \geq 1)$, and $\mathrm{IE}_{0}=$ IOpen. Also, two types of initial segments are defined, and finally, a brief introduction on $\beta M$ (being $M$ a semigroup) will be shown, focusing on its algebraic and topological features.

\subsection{Subsystems of $\mathrm{I} \Delta_{0}$}

The language of the Arithmetic is $L=\{+, \cdot,<, 0,1\}$. Quantifications like $\exists x(x<t \wedge \varphi)$ or $\forall x(x<t \rightarrow \varphi)$ (denoted by $\exists x<t \varphi$ and $\forall x<t \varphi$ ) are called bounded. 
The set $\Delta_{0}$ is the set of bounded formulas (which have only bounded quantifiers, respectively). Similar to the definitions of the classical fragments in Peano Arithmetic PA, the classes $\mathrm{E}_{n}, \mathrm{U}_{n}$ are defined according to the alternation of (bounded) quantifiers:

$$
\begin{aligned}
& \mathrm{E}_{0}\left(=\mathrm{U}_{0}\right) \text { is the class of open formulas, } \\
& \mathrm{E}_{n+1}:=\left\{\exists x_{1}<t_{1} \cdots \exists x_{m}<t_{m} \varphi \mid \varphi \in \mathrm{U}_{n}\right\}, \text { and } \\
& \mathrm{U}_{n+1}:=\left\{\forall x_{1}<t_{1} \cdots \forall x_{p}<t_{p} \varphi \mid \varphi \in \mathrm{E}_{n}\right\}
\end{aligned}
$$

(with $t_{i} L$-terms). Therefore $\Delta_{0}=\mathrm{E}_{\omega}=\bigcup_{k} \mathrm{E}_{k}$.

The (finite) theory $\mathrm{PA}^{-}$has as models the nonnegative part of discretely ordered rings.

The paper will work on the well-known Induction (I), Least element (L) and Collection (B) schemas, and the theories

$$
\mathrm{E} \Gamma=\mathrm{PA}^{-}+\left\{\mathrm{E}_{\varphi} \mid \varphi \in \Gamma\right\}
$$

where $\mathrm{E}=\mathrm{I}, \mathrm{L}, \mathrm{B}$ and $\Gamma=\mathrm{E}_{n}, \mathrm{U}_{n}$ (see [30] and [51]). It is unknown whether $\left\{\mathrm{IE}_{n}\right\}_{n<\omega}$ collapses. Only partial results are known. Among them, the following ones:

- $\mathrm{IE}_{0} \equiv \mathrm{LE}_{0}, \mathrm{IE}_{0} \nvdash \mathrm{IE}_{1}([43])$.

- $\mathrm{IE}_{0}$ plus the full collection scheme does not prove $\mathrm{IE}_{1}[3]$.

- $\mathrm{IE}_{n+1} \Longleftrightarrow \mathrm{LU}_{n} \Longrightarrow \mathrm{IE}_{n} \Longleftrightarrow \mathrm{IU}_{n} \Longleftrightarrow \operatorname{LE}_{n}(n \geq 1$; see [28,51]).

Another useful scheme for the paper is the following one:

$$
\mathrm{G}_{\psi}(x):=\forall \vec{v}<x[\exists u<x \psi(u, \vec{v}) \rightarrow \exists u<x(\psi(u, \vec{v}) \wedge \forall w<u \neg \psi(w, \vec{v}))] .
$$

It is easy to see that

$$
\mathrm{PA}^{-}+\left\{\forall u \mathrm{G}_{\psi}(u): \psi \in \mathrm{E}_{k}\right\}
$$

is an alternative axiomatization of $\mathrm{IE}_{k}$.

\subsection{Open induction and subsystems}

If models of $\mathrm{IE}_{0}$ are considered, then a large number of results about their algebraic nature can be proved. A seminal paper on this topic was [43], where J. C. Shepherdson shows the following (recursive) nonstandard model $\mathbb{N}_{X} \models \mathrm{IE}_{0}$. Let $K$ be the field of real algebraic numbers and $X$ an indeterminate. The universe of $\mathbb{N}_{X}$ is the set of polynomials

$$
\left\{a_{p} X^{p / q}+\cdots+a_{1} X^{1 / q}+a_{0}: a_{p}, \ldots, a_{1} \in K, a_{p}>0, a_{0} \in \mathbb{Z}, p \in \mathbb{N}, q \in \mathbb{N}^{*}\right\},
$$

the addition and multiplication are naturally defined, and the order " $<$ " is interpreted as: $P(X)<Q(X)$ if the leader coefficient of $Q(X)-P(X)$ is $>0$.

Shepherdson's construction will be generalized (in the first part of the paper) to some kind of extensions and models. The most direct one is to replicate it to obtain, given any $M \models \mathrm{IE}_{0}$, an end-extension $M \subset_{e}$ $M_{X} \models \mathrm{IE}_{0}$ (The model thus constructed will be called Shepherdson's extension of $M$ ).

An algebraic characterization of $\mathrm{IE}_{0}$ (also proved in [43]) is the following:

Result 2.1. The models of $\mathrm{IE}_{0}$ are those models of $\mathrm{PA}^{-}$that are the integer part of their real algebraic closure. 
See [21] for other analogous results that provide algebraic theories equivalent to various fragments of $\mathrm{IE}_{0}$.

Please note also that Shepherdson's algebraic construction produces a recursive model if a recursive one is extended. Thus it is not useful to build (extensions of) of models of $\mathrm{IE}_{1}$, due to Tennenbaum phenomena in $\mathrm{IE}_{1}$ :

Result 2.2. There are no recursive nonstandard models of $\mathrm{IE}_{1}[51]$.

Shepherdson's work illustrates how the construction of real closed fields can be related to obtaining adequate extensions of the fraction field of an arithmetic model [12]. See also the more recent paper [46], where Tanaka and Tsuboi present a new construction of real closed fields using an elementary extension of an ordered field that has an integer part that satisfies PA. It is also interesting to review the relationship obtained by D'Aquino, Knight and Strachencko in [11], where it is shown that the real closure of a model I $\Sigma_{4}$ is recursively saturated, thus establishing a relationship between a model-theoretic property of (ordered) fields and classical induction schemes on Arithmetic. Alternatively, it is possible to study the inverse path, that is, to analyze the real closed fields that admit an integer part whose non-negative part is a model of PA (see [6]).

Throughout the paper, two remarkable subsystems of $\mathrm{IE}_{0}$ are considered, which will be used as base theories. The theory $\mathrm{IE}_{0}^{1}$ augments $\mathrm{PA}^{-}$with the induction scheme for open formulas whose atomic subformulas are linear inequalities. The theory is equivalent to

$$
\mathrm{PA}^{-}+\{\exists u(v u \leq x<v(u+1))\}
$$

(i.e. $\mathrm{PA}^{-}$plus Euclidean division), and it is strictly weaker than $\mathrm{IE}_{0}$. The theory of integer roots,

$$
\mathrm{RIE}_{0}^{1}:=\mathrm{IE}_{0}^{1}+\left\{\exists u\left(u^{k} \leq x<u^{k+1}\right): k<\omega\right\}
$$

is also weaker than $\mathrm{IE}_{0}$. Moreover, the extension $\mathrm{IE}_{0}^{1} \subset \mathrm{RIE}_{0}^{1}$ is proper (all these results can be found in $[5])$.

\subsection{Exponentiation and segments determined by (standard) powers or integer roots}

Let us suppose $\mathrm{PA}^{-} \subseteq T$ and $\mathbb{N} \models T$. A function $f: \omega^{n} \rightarrow \omega$ is provably recursive in $T$ if there is $\theta(\vec{x}, y, z) \in \Delta_{0}$ such that $\exists z \theta(\vec{x}, y, z)$ defines the graph of $f$ and $T \vdash \forall \vec{x} \exists ! y \exists z \theta(\vec{x}, y, z)$. Such functions have been characterized for strong fragments (cf. [22]).

According to Result 1.1 the function $(x, y) \mapsto x^{y}$ is not provably recursive in $\mathrm{I} \Delta_{0}$, although there is a bounded formula $\eta(x, y, z) \in \Delta_{0}$ that represents this function in $\mathrm{I} \Delta_{0}$ and such that the basic properties are provable in $\mathrm{I} \Delta_{0}$ (see e.g. [20]). To simplify the following notation is introduced: $x^{y}=z$ will mean $\eta(x, y, z)$, and $\exp$ is the sentence $\forall x \forall y \exists z\left(x^{y}=z\right)$.

Remark 2.3. Throughout the paper, $k_{e}$ will be an integer such that $\mathrm{IE}_{k_{e}}$ proves the basic properties of $x^{y}=z$ as well as the weak overspill principle for $\mathrm{U}_{m+1}$-formulas, for some $m$ such that $x^{y}=z \in \mathrm{E}_{m}$.

Two kinds of segments are used in this paper. The segment determined by standard powers of $a \in M \models$ $\mathrm{PA}^{-}$is

$$
a^{\omega}:=\left\{b \in M: \exists n<\omega\left(b<a^{n}\right)\right\}
$$

It is straightforward to prove that 


$$
a^{\omega}=\bigcap\left\{N \subseteq_{e} M: N \models \mathrm{PA}^{-} \text {and } a \in N\right\}
$$

When $a \notin \omega$, it is defined the segment bounded by the integer standard roots of $a$ as

$$
a^{\frac{1}{\omega}}:=\left\{b \in M: \forall n<\omega\left(b^{n}<a\right)\right\}
$$

for which it can be shown that

$$
a^{\frac{1}{\omega}}=\bigcup\left\{N \subset_{e} M: N \models \mathrm{PA}^{-} \text {and } a \notin N\right\} .
$$

Notation: As usual, it will be written $\varphi(M)$ instead of $\{a \in M: M \models \varphi(a)\}$, and $L(M)$ denotes the language $L$ augmented by constants denoting the elements of $M$. To simplify notation, it will be written " $M \models T_{1}+\neg T_{2}$ " to mean " $M \models T_{1}$ and $M \not \models T_{2}$ ".

\subsection{Algebra on Stone-Čech compactification of a (discrete) semigroup}

Let us recall that Stone-Čech compactification $\beta S$ of a discrete space $S$ is the compact Hausdorff space whose elements are the ultrafilters on $S$ (being the elements of $S$ identified with the principal ones). A basis of the topology is given by the (clopen) sets

$$
\bar{A}=\{p \in \beta S: A \in p\} \text { for each } A \subseteq S
$$

The set $S$ is dense in $\beta S$. It will be denoted by $S^{*}$ to the set $\beta S \backslash S$.

The construction is functorial in nature, i.e. for each function $f: S \rightarrow S$ induces a (unique) extension $\beta f: \beta S \rightarrow \beta S$ such that the following diagram is commutative:

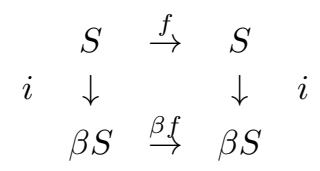

The extension $\beta f$ is defined by

$$
\beta f(p):=\left\{A \subseteq S: f^{-1}(A) \in p\right\}
$$

The operation $*$ of a discrete semigroup $\left(M, *_{M}\right)$ is extended to $\beta M$ as follows (cf. [24], chap. 4, sect. 1): If $p, q \in \beta M$, the ultrafilter $p * q$ is defined by:

$$
A \in p * q \text { if and only if }\{x:\{y: x * y \in A\} \in q\} \in p .
$$

The structure $(\beta M, *)$ is a (compact) right-topological semigroup. An excellent reference for this topic is the exposition [24].

The idempotent elements of such a semigroup are especially interesting for our purposes. A result that assures its existence is the following:

Result 2.4. [17] Every compact right topological semigroup has an idempotent element.

The interest in these elements lies in their combinatorial reading, since they determine a certain Ramseylike regularity. Let us formalize such a property. 
A $F P$-set in a semigroup $(S, \cdot)$ is a set which contains all the finite products of a set, i.e. a subset like

$$
F P\left(\left\{x_{n}\right\}_{n<\omega}\right):=\left\{\Pi_{n \in F} x_{n}: F \subset \omega \text { and }|F|<\aleph_{0}\right\},
$$

for some $\left\{x_{n}\right\}_{n<\omega}$ sequence (in additive notation, $F S\left(\left\{x_{n}\right\}_{n<\omega}\right)$ ). A version of Galvin-Glazer theorem of the Finite Sums Theorem, in terms of idempotents elements, is the following:

Result 2.5. (see e.g. Th. 5.8 of [24]) If $A \in p$ and $p$ is a nonprincipal idempotent ultrafilter in $(\beta M, \cdot)$, then $A$ is a FP-set.

In the second part of the paper the following property on the fixed points will be used.

Result 2.6. (see e.g. Th. 3.35 in [24]) Let $\beta f: \beta M \rightarrow \beta M$. If $p \in \beta M$ is a fixed point for $\beta f$ then

$$
\{x \in M: f(x)=x\} \in p
$$

For example, consider $\beta f: \beta M \rightarrow \beta M$ (being $M \models \mathrm{PA}^{-}$) be the extension of $f(x):=x^{2}$. By above result it has not any fixed point in $\beta M \backslash\{0,1\}$.

It is also possible to generalize the composition of $\beta f$ as follows: given a ultrafilter $r \in \omega^{*}$ (recall that $\left.\omega^{*}=\beta \omega \backslash \omega\right)$, it is defined the $r$-iteration of $\beta f$ as

$$
(\beta f)^{r}(p)=q \quad \Longleftrightarrow\left\{n:(\beta f)^{n}(p)=q\right\} \in r
$$

this type of iteration is very useful because of the following property:

Theorem 2.7. (folklore, see e.g. [2]) Let $(X, f)$ be a topological dynamic system (thus $X$ is compact). The following conditions are equivalent:

1. $x \in X$ is recurrent (that is, for any $U$ neighborhood in $X$ the set $\left\{n \in \omega: f^{n}(x) \in U\right\}$ is infinite)

2. There exists $r \in \omega^{*}$ such that $f^{r}(x)=x$

Therefore, since recurrent points exist, there also exists $r \in \mathbb{N}^{*}$ such that $f^{r}(x)=x$ for some $x \in X$. Particularize us this fact for our case. If the dynamic system $(\beta M, \beta f)$ is considered, it can conclude that

Result 2.8. For any $f: M \rightarrow M$ there exist $r \in \mathbb{N}^{*}$ and $p \in \beta N$ such that $(\beta f)^{r}(p)=p$.

\section{Part I: maximal segments, kernels and gaps}

The initial segments that will be used in the rest of the article are studied in this section.

Definition 3.1. [3] Let $M \models \mathrm{PA}^{-}$and $T \subseteq \operatorname{Th}(\mathbb{N})$. The $T$-kernel of $M$ is

$$
M(T):=\bigcup\{I \subseteq e M \mid I \models T\}
$$

Since $M(T)$ is the union of an increasing chain of initial segments, if $T$ is $\Pi_{1}$-axiomatizable (for example $\left.\mathrm{IE}_{n}\right)$ then $M(T) \models T$.

Given a formula $\varphi(x, \vec{a})$, the notation

$$
\varphi(x, \vec{a})<u
$$

(or simply $\varphi<u$ ) will be used to mean that $\exists v \leq u \varphi(v, \vec{a})$, and $u<\varphi$ means $\neg(\varphi<u)$. 
Definition 3.2. Let $M \models \mathrm{PA}^{-}$. An $L(M)$-formula $\varphi(x)$ is said a gap in $M$ if $M \not \models \mathrm{L}_{\varphi}$.

The following lemma is a very useful characterization of the elements that do not belong to a $\mathrm{IE}_{k}$-kernel:

Lemma 3.3. Let $M \models \mathrm{PA}^{-}$. If $a \in M \backslash M\left(\mathrm{IE}_{k}\right)(0 \leq k \leq \omega)$ then there exists $n \in \omega$ and $\psi$ an $E_{k}$-gap in $M$ with parameters in $a^{\omega}$ such that $M \models \psi<a^{n}$. Moreover, if $M \models \mathrm{IE}_{0}^{1}$ then it can be selected $\psi$ with parameters in $[0, a]$.

Proof. If $a \notin M\left(\mathrm{IE}_{k}\right)$ then $a^{\omega} \not \models \mathrm{IE}_{k}$. Thus is $\psi(x, \vec{b}) \in \mathrm{E}_{k}$ with $\vec{b} \in a^{\omega}$, such that $a^{\omega} \not \forall \mathrm{L}_{\psi}$.

The additional property for $\mathrm{IE}_{0}^{1}$-models is easy to justify, by using Euclidean division on the parameters.

Remark 3.4. It is easy to prove, using the above lemma, that $M(\mathrm{PA})$ is nonstandard in every $\Delta_{0}$-recursively saturated model of $\mathrm{PA}^{-}$. This fact is true because the following type can be realized:

$$
\mathbf{p}(v):=\left\{m<v \wedge \forall u<v^{m} \mathrm{G}_{\varphi_{m}}(u): m \in \omega\right\}
$$

(where $\left\{\varphi_{m}\right\}_{m<\omega}$ is a recursive enumeration of $\mathrm{E}_{1}$ ). The element that realizes $\mathbf{p}(v)$ belongs to $N=M\left(\mathrm{IE}_{1}\right)$, so this segment is nonstandard. According to Paris' result given in [42] (see also [29]), $N(\mathrm{PA})$ (which coincides with $M(\mathrm{PA}))$ is nonstandard.

In fact, it is sufficient to have $\mathrm{U}_{3}$-recursive saturation.

From now on it will be studied some relationships between the $T$-kernels and the initial segments defined in Sect. 2.3. In the case of Shepherdson's model, $\mathbb{N}_{X}$ is satisfied that

$$
2^{\omega}=X^{\frac{1}{\omega}}=\mathbb{N}=\mathbb{N}_{X}\left(\mathrm{I} \Delta_{0}\right)
$$

In general, if $M$ is a model of a weak theory and $a \in M \backslash M\left(\mathrm{IE}_{k}\right)$, then $M\left(\mathrm{IE}_{k}\right) \subseteq_{e} a^{\frac{1}{\omega}}$. Likewise, if $a \in M\left(\mathrm{IE}_{k}\right)$, then $a^{\omega} \subseteq M\left(\mathrm{IE}_{k}\right)$. Therefore, it can be "approximated" the $\mathrm{IE}_{k}$-kernels with that kind of initial segments. Such an approach can either achieve equality or at least to be arbitrarily approximate, by properly choosing elements. This fact suggests the following definition, where a name is given to the possible relationships between both types of segments.

Definition 3.5. Let $M \models \mathrm{PA}^{-}$, and $0 \leq k \leq \omega$. It is said that:

1. $M$ is $k$-short if there is $a \in M$ such that $a^{\omega}=M\left(\mathrm{IE}_{k}\right)$. Otherwise it is said that $M$ is $k$-long.

2. $M$ is $k$-high if there is $a \in M$ such that $a^{\frac{1}{\omega}}=M\left(\mathrm{IE}_{k}\right)$. Otherwise it is said that $M$ is $k$-low.

Throughout the following sections, several relations between those kinds of segments and other problems on Weak Arithmetics will be proven (they are described in Fig. 2). Several of them will be used or refined in the second part of the paper.

By generalizing Shepherdson's construction, several examples of the models defined above can be shown (see Table 1):

- Shepherdson's extension of a model of $\mathrm{IE}_{k}$ is $k$-high $(1 \leq k \leq \omega)$, and $\mathbb{N}_{X}$ is $\omega$-short.

- A $\omega$-low model of $\mathrm{IE}_{0}$ is $M_{\downarrow}:=\bigcup_{i<\omega} M_{i}$, where

$$
M_{n}=\left(\cdots\left(\mathbb{N}_{X_{n}}\right)_{X_{n-1}} \cdots\right)_{X_{0}}
$$




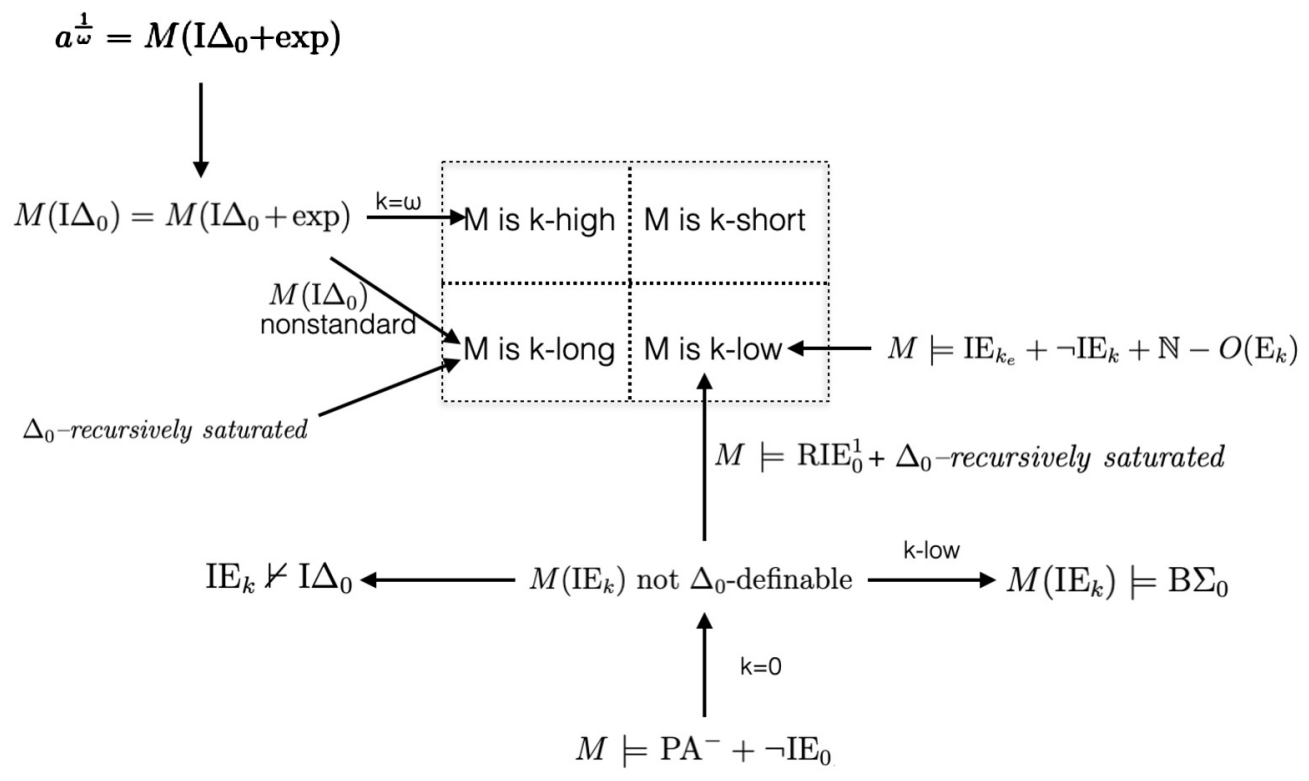

Fig. 2. Results about kernels (for models of $\mathrm{PA}^{-}$).

Table 1

Examples of initial segments.

\begin{tabular}{lll}
\hline & $k$-high & $k$-low \\
\hline$k$-short & $\mathbb{N}_{X}$ & $M_{\downarrow}$ \\
$k$-long & $M_{X}, M \models \mathrm{I} \Delta_{0}+\exp$ & Corollary 3.9 \\
\hline
\end{tabular}

( $M_{\downarrow}$ is an integer part of its real closure because this closure satisfies $K^{M_{\downarrow}} \cong \bigcup_{i<\omega} K^{M_{i}}$, hence $M_{\downarrow} \models$ $\left.\mathrm{IE}_{0}\right)$, and $M_{\downarrow}\left(\mathrm{I} \Delta_{0}\right)=\mathbb{N}$.

For stronger theories the following result holds:

Proposition 3.6. Let $M \models P A^{-}$If

$$
a^{\frac{1}{\omega}}=M\left(\mathrm{I} \Delta_{0}+\exp \right)
$$

for some a, then

$$
M\left(\mathrm{I} \Delta_{0}\right)=M\left(\mathrm{I} \Delta_{0}+\exp \right)
$$

(thus $M$ is $\omega$-high and, if $M\left(\mathrm{I} \Delta_{0}\right)$ is nonstandard, $\omega$-long).

Proof. Otherwise, if $a \in M\left(\mathrm{I} \Delta_{0}\right) \backslash M\left(\mathrm{I} \Delta_{0}+\right.$ exp), it is sufficient to apply $\Delta_{0}$-overspill in $M$ (I $\left.\Delta_{0}\right)$ on the $\Delta_{0}$-formula $\gamma(v) \equiv \exists u<a\left(v^{v}=u\right)$ to reach a contradiction.

\subsection{Definability of kernels}

For stronger theories the following result holds: The problem of $\Delta_{0}$-definability of $\mathrm{IE}_{k}$-kernels is related to the collection schema. It holds that if $M \models \mathrm{IE}_{k+1}+\neg \mathrm{IE}_{k+2}$, then $M\left(\mathrm{IE}_{k+2}\right) \models \mathrm{BE}_{k}[3]$.

Proposition 3.7. If $M\left(\mathrm{IE}_{k}\right) \not \models \mathrm{B} \Sigma_{0}$ then it is $\Delta_{0}$-definable. 
Proof. Let $\varphi(x, y)$ be a $L(M)$-formula such that $M\left(\mathrm{IE}_{k}\right) \not \models \mathrm{B}_{\varphi}$, that is

$$
M\left(\mathrm{IE}_{k}\right) \models \forall x \leq b \exists y \varphi(x, y) \wedge \forall z \exists x \leq b \forall y<z \neg \varphi(x, y)
$$

then the formula $\exists x \leq b \forall y<z \neg \varphi(x, y)$ defines $M\left(\mathrm{IE}_{k}\right)$ in $M$.

In $\Delta_{0}$-recursively saturated models, properties of kernels and $\Delta_{0}$-definability issues are related. Please let us note that in bounded induction, $\Delta_{0}$-recursive saturation has a different behavior than in strong fragments (see e.g. [9], Sect. 2). It is known that if $a \in M\left(\mathrm{I} \Delta_{0}\right)$ and $2^{a^{\omega}} \subsetneq M\left(\mathrm{I} \Delta_{0}\right)$ then the relational structure $[0, a]$ is recursively saturated [37]. Therefore, if $M\left(\mathrm{I} \Delta_{0}+\exp \right)=M\left(\mathrm{I} \Delta_{0}\right)$, then $M\left(\mathrm{I} \Delta_{0}\right)$ is short recursively saturated. This kind of models presents nice properties (see e.g. [18] for an exposition) Let us first see some results on this type of model.

Theorem 3.8. Let $M \models \mathrm{PA}^{-}$be $\Delta_{0}$-recursively saturated and $0 \leq k \leq \omega$. It holds that:

1. $M$ is $k$-long.

2. If $M \models \mathrm{RIE}_{0}^{1}$ verifies that $M\left(\mathrm{IE}_{k}\right)$ is $\Delta_{0}$-definable, then $M$ is $k$-low. This fact is not true in general for models of $\mathrm{IE}_{0}^{1}$.

Proof. To prove (1), consider $\left\{\varphi_{i}: i \in \omega\right\}$ a recursive enumeration of $\mathrm{E}_{k}$ and $b \in M\left(\mathrm{IE}_{k}\right)$. Then

$$
\mathbf{p}(v ; b)=\left\{v>b^{n} \wedge \bigwedge_{1 \leq i \leq n} \mathrm{G}_{\varphi_{i}}\left(v^{m}\right): n, m<\omega\right\}
$$

$\left(v^{m}\right.$ is the term $\left.v^{(m)} \cdot v\right)$ is a finitely satisfiable type in $M$. Any element realizing $\mathbf{p}(v)$ belongs to $M\left(\mathrm{IE}_{k}\right) \backslash b^{\omega}$

To prove (2), assume that $a \in M \models \operatorname{RIE}_{0}^{1}$ verifies $a^{\frac{1}{\omega}}=M\left(\mathrm{IE}_{k}\right)$. If $\varphi \in \Delta_{0}$ defines $M\left(\mathrm{IE}_{k}\right)$, then the type

$$
\mathbf{p}(v ; a)=\{\neg \varphi(v)\} \cup\left\{v^{n}<a: n \in \omega\right\}
$$

is finitely satisfiable. Contradiction.

To conclude the proof, it remains to see that 3.8.(2) cannot be improved to $\mathrm{IE}_{0}^{1}$, by giving a countermodel:

Let $M_{1}$ be the structure whose universe is

$$
\left\{a_{n} X^{n}+\cdots+a_{1} X+a_{0}: a_{i} \in \mathbb{Q}, a_{n}>0, a_{0} \in \mathbb{Z} \text { if } n>0 \text { or } a_{0} \in \mathbb{N} \text { if } n=0\right\}
$$

The addition, product and order relation are defined as usual. It verifies that $M_{1} \models \mathrm{IE}_{0}^{1}[5]$.

A first property of $M_{1}$ is that the set $\omega$ is defined by the open formula

$$
\varphi(u) \equiv u^{2}<X
$$

Let $M$ be a $\Delta_{0}$-recursively saturated elementary extension of $M_{1}$. The following results hold:

(i) For all $n \geq 2$ it is true that $M \models u^{n}<X \leftrightarrow u^{2}<X$.

(ii) For each $\psi(u, \vec{y}) \in \Delta_{0}$

$$
M \models \forall \vec{y}\left[\exists u\left(u^{2}<X \wedge \psi(u, \vec{y})\right) \rightarrow \exists v(\psi(v, \vec{y}) \wedge \forall w<v \neg \psi(w, \vec{y}))\right]
$$

(iii) $M \models \neg \varphi(u) \rightarrow \neg \mathrm{G}_{\neg \varphi}(u)$. 
From $(i),(i i)$ and $(i i i)$ it is concluded that $X^{\frac{1}{\omega}}=M\left(\mathrm{I} \Delta_{0}\right)$, but $M\left(\mathrm{I} \Delta_{0}\right)$ is definable by $\varphi(u)$, an $E_{0}$ formula.

Analogously countermodels for finite fragments of $\mathrm{RIE}_{0}^{1}$ can be found, using similar models which are introduced in [5].

Corollary 3.9. Let $M \models \mathrm{IE}_{k}$. Every $\Delta_{0}$-recursively saturated elementary extension $M^{\prime}$ of the Shepherdson extension $M_{X}$ is $k$-low and $k$-long.

Proof. $M^{\prime}$ is $k$-long by Theorem 3.8(1). Moreover $M$ is $k$-low as consequence of 3.8.(2): the formula

$$
\varphi(u) \equiv u=0 \vee(u|X \wedge(u+1)| X)
$$

defines $M^{\prime}\left(\mathrm{IE}_{k}\right)$ in $M^{\prime}$ (if $M \models \mathrm{IE}_{k}$ ). This is true because the following formulas are true in $M^{\prime}$ (they are true in $M)$ :

- $M^{\prime} \models \neg \varphi(u) \rightarrow \neg \mathrm{G}_{\neg \varphi}(u)$

- $M^{\prime} \models \varphi(u) \rightarrow \mathrm{G}_{\psi}(u)$ for each $\psi \in E_{k}$

The analysis made in Theorem 3.8 can be supplemented by outlining the hardness of deciding whether the reciprocal of 3.8.(2) holds: if such reciprocal fails, then $\mathrm{IE}_{k} \nvdash \mathrm{I} \Delta_{0}$.

Theorem 3.10. If there is a k-low model $M \models \mathrm{RIE}_{0}^{1}$ with $M\left(\mathrm{IE}_{k}\right)$ not $\Delta_{0}$-definable, then $\mathrm{IE}_{k} \nvdash \mathrm{I} \Delta_{0}$.

Proof. Suppose that there is such a model $M$ and $\mathrm{IE}_{k} \vdash \mathrm{I} \Delta_{0}$. By Result 1.3 one would conclude that $\mathrm{IE}_{k}$ is finitely axiomatizable; so there are $\mathrm{E}_{k}-$ formulas $\varphi_{1}, \ldots, \varphi_{n}$ such that $\mathrm{IE}_{k}$ is equivalent to the theory

$$
\mathrm{PA}^{-}+\left\{\forall u \mathrm{G}_{\varphi_{1}}(u), \ldots, \forall u \mathrm{G}_{\varphi_{n}}(u)\right\}
$$

Then the following formula defines $M\left(\mathrm{IE}_{k}\right)$ :

$$
\gamma(u):=\bigwedge_{1 \leq i \leq n} \mathrm{G}_{\varphi_{i}}(u)
$$

This is true because if $M \models \gamma(b)$, then $b^{\frac{1}{\omega}} \subsetneq M\left(\mathrm{IE}_{k}\right)\left(M\right.$ is $\omega$-low), so $b \in M\left(\mathrm{IE}_{k}\right)$.

This theorem will be improved later (Corollary 3.24). Please let us note that the $\omega$-low model $M_{\downarrow}$, described before would not be a candidate to apply the theorem above: $M_{\downarrow}\left(\mathrm{IE}_{k}\right)$ is definable in $M_{\downarrow}$ by

$$
\psi(u) \equiv \forall v<u\left(v=0 \vee \forall y \leq v\left(v^{2} \neq 2 y^{2}\right)\right)
$$

which has only parameters in $M_{\downarrow}\left(\mathrm{IE}_{k}\right)=\mathbb{N}$.

In Shepherdson's model $\mathbb{N}_{X}\left(\mathrm{IE}_{1}\right)=\mathbb{N}$ holds, being the segment definable by the formula $\psi(u)$ above. It is known that $\psi(u) \in \mathrm{E}_{1}$ in $\mathrm{IE}_{0}$ (see [3]). It is interesting to note that this kind of characterization is not possible for $M\left(\mathrm{IE}_{0}\right)$ if it is only required to be a model of $\mathrm{PA}^{-}$:

Proposition 3.11. If $M \models \mathrm{PA}^{-}+\neg \mathrm{IE}_{0}$, then $M\left(\mathrm{IE}_{0}\right)$ is not $\mathrm{E}_{0}$-definable in $M$ with parameters in the own segment. 
Proof. Let us assume otherwise, that there exists $\varphi(x, \vec{u}) \in \mathrm{E}_{0}$ and $\vec{a} \in M\left(\mathrm{IE}_{0}\right)$ such that $\varphi(x, \vec{a})$ defines $M\left(\mathrm{IE}_{0}\right)$.

Let $K^{M}$ be the real closure of $M$ (by Result $2.1 M$ is not integer part of $K^{M}$, because $M \not \models \mathrm{IE}_{0}$ ), and $K^{\prime}$ the real closure of $M\left(\mathrm{IE}_{0}\right)$ within $K^{M}$. Since $M\left(\mathrm{IE}_{0}\right)$ is a whole part of their actual closure, it verifies that

$$
K^{\prime} \models \forall x \exists y(x \geq 0 \rightarrow y \leq x<y+1 \wedge \varphi(y, \vec{a}))
$$

By model completeness of the theory of real closed fields, $K^{\prime} \prec K^{M}$, so $K^{M}$ satisfies $(*)$. Then $M\left(\mathrm{IE}_{0}\right)$ would be an integer part in $K^{M}$, which is a contradiction.

\subsection{Collapse of kernels and interpretability}

Once the definability of the kernels has been examined, the next concern to be studied is about two related questions. The first one asks whether there exists a model with internal structure like Shepherdson's model (that is, $k$-high and $k$-short for every $k$ ) but for infinite and different kernels, (that would imply that $\mathrm{I} \Delta_{0}$ is not finitely axiomatizable). It will be seen that there is no such model under some assumptions (Corollary 3.15): in an $\omega$-high and $\omega$-short model the hierarchy of kernels $\left\{M\left(\mathrm{IE}_{k}\right)\right\}_{k<\omega}$ collapses. Please note that this fact does not imply that $\left\{\mathrm{IE}_{n}\right\}_{n}$ collapses, nor that $\mathrm{I} \Delta_{0}$ is finitely axiomatizable modulo $\mathrm{IE}_{0}$, but it suggests the second question:

Open Problem 3.12. Is $\mathrm{I} \Delta_{0}$ a theory $\Pi_{1}$-interpretable in $\mathrm{IE}_{0} ?$

The aim will be to demonstrate on that issue that $\mathrm{I} \Delta_{0}$ has certain maximal status as possible $\Pi_{1}$-interpretable theory on $\mathrm{RIE}_{0}^{1}$. In what follows it will be used the index $k_{e}$ introduced in Remark 2.3.

Firstly it is going to be related to the collapse of $\left\{\mathrm{IE}_{n}\right\}_{n<\omega}$ with a problem on $\Delta_{0}$-elementary extensions:

Theorem 3.13. Suppose that every model of $\mathrm{IE}_{k_{e}}$ has a $\Delta_{0}$-elementary extension model of $\mathrm{RIE}_{0}^{1}$, which is $\omega$-high and $\omega$-short. Then $\left\{\mathrm{IE}_{k}\right\}_{k<\omega}$ collapses.

Proof. Let us see that $\mathrm{IE}_{k_{e}} \vdash \mathrm{I} \Delta_{0}$, being $k_{e}$ any index satisfying the features required in Remark 2.3. All one needs to do is prove that if $N \models \mathrm{IE}_{k_{e}}$ then $N \models \mathrm{I} \Delta_{0}$.

Let $N$ be such a model, and let $M \models \operatorname{RIE}_{0}^{1}$ be a $\Delta_{0}$-elementary extension of $N$ which is $\omega$-high and $\omega$-short.

Claim: $M\left(\mathrm{IE}_{k_{e}}\right)=M\left(\mathrm{I}_{0}\right)$.

Proof of the claim: Assume that there is $a \in M\left(\mathrm{IE}_{k_{e}}\right) \backslash M\left(\mathrm{I} \Delta_{0}\right)$. Let $b \in M\left(\mathrm{I} \Delta_{0}\right)$ and

$$
\psi(u) \equiv \forall x<u \exists z<a\left(b^{x}=z \wedge z^{x}<a\right) .
$$

It holds that $M\left(\mathrm{IE}_{k_{e}}\right) \models \psi(n)$ for each $n \in \omega$. By weak $\mathrm{U}_{m+1^{-}}$overspill, there is $\delta \in M\left(\mathrm{I} \Delta_{0}\right) \backslash \omega$ such that $M \models \psi(\delta)$. Consider now the model of $\mathrm{PA}^{-}$:

$$
I=\left\{d \in M: \exists \alpha \in \delta^{\frac{1}{\omega}}\left(d<b^{\alpha}\right)\right\}
$$

It is straightforward to prove that $I \models P A^{-}$and $b^{\omega} \subsetneq I \subseteq a^{\frac{1}{\omega}}\left(M\left(\mathrm{I} \Delta_{0}\right)\right.$ is nonstandard, so $\delta^{\frac{1}{\omega}} \neq \omega$ because the model is $\omega$-high). Therefore, $b^{\omega} \neq \mathcal{M}\left(\mathrm{I} \Delta_{0}\right)$, so $M$ is $\omega$-long. Contradiction.

The claim, together with the fact that $N \subseteq M\left(\mathrm{IE}_{k_{e}}\right)=M\left(\mathrm{I} \Delta_{0}\right)$, implies that for each $a \in N$ and $\varphi(x) \in \Delta_{0}$ it has $N \models \mathrm{G}_{\varphi}(a)$ (because $\left.M \models \mathrm{G}_{\varphi}(a)\right)$. Therefore, $N \models \mathrm{I} \Delta_{0}$. 
Notice that in the demonstration of Theorem 3.13, the following result has been demonstrated, which we believe to be of interest in itself.

Corollary 3.14. Let $M \models \mathrm{RIE}_{0}^{1}$ be such that $M\left(\mathrm{I} \Delta_{0}\right)=a^{\frac{1}{\omega}}=b^{\omega} \neq \mathbb{N}$ for some $a, b \in M$. Then $M\left(\mathrm{IE}_{k}\right)=$ $M\left(\mathrm{I} \Delta_{0}\right)$ for some $k$.

An interesting question about the special status of $\mathrm{IE}_{1}$ in the hierarchy $\left\{\mathrm{IE}_{n}\right\}_{n}$, is whether Corollary 3.14 is true for $k=1$, since for $k=0$ does not hold. Let us note that, by Theorem 3.13, there is not $M \models \mathrm{IE}_{0}$ with a stratification like Shepherdson's model for infinite kernels.

Corollary 3.15. There is $k \geq 1$ such that for each $\omega$-high $M \models \mathrm{RIE}_{0}^{1}$ with $M\left(\mathrm{I}_{0}\right)$ nonstandard, one of the following conditions is true:

1. $M\left(\mathrm{IE}_{k}\right)=M\left(\mathrm{I} \Delta_{0}\right)$.

2. $M$ is $\omega$-long.

Proof. Similar to that of 3.13 , taking $k=k_{e}$.

Please note that Corollary 3.15 is already independent of the question of whether $\left\{\mathrm{IE}_{n}\right\}_{n<\omega}$ collapses. The plausible collapse of $\mathrm{IE}_{k}$-kernels suggests that $\mathrm{I} \Delta_{0}$ may be $\Pi_{1}$-interpretable in a class of models of $\mathrm{RIE}_{0}^{1}$ (recall that if $\mathrm{IE}_{k} \vdash \mathrm{I} \Delta_{0}$ then $\mathrm{I} \Delta_{0}$ is finitely axiomatizable). This issue will be examined below.

Definition 3.16. A $\Pi_{1}$-formula $\Phi(u)$ interprets $\mathbf{I} \Delta_{0}$ in a theory $\mathrm{T}$ if $\Phi$ defines an initial segment which is a model of $\mathrm{I} \Delta_{0}$, that is:

- $\mathrm{T} \vdash \Phi(0) \wedge \forall x, y(x \leq y \wedge \Phi(y) \rightarrow \Phi(x))$.

- $\mathrm{T} \vdash \forall x, y(\Phi(x) \wedge \Phi(y) \rightarrow \Phi(x \cdot y) \wedge \Phi(x+1) \wedge \Phi(x+y))$.

- For each $\psi \in \Delta_{0}, \mathrm{~T} \vdash \forall x\left(\Phi(x) \rightarrow \mathrm{G}_{\psi}(x)\right)$ (hence $\left.\mathrm{T}+\forall u \Phi(u) \vdash \mathrm{I} \Delta_{0}\right)$.

See [49] for some results about interpretability of theories in models of I $\Delta_{0}$.

The following result can be useful to analyze the question of whether $\mathrm{I}_{0}$ is $\Pi_{1}$-interpretable in $\mathrm{RIE}_{0}^{1}$. (Please let us compare the next theorem with Th. 5.26 in chap. V of [22], as well as their proofs):

Theorem 3.17. Let $\mathrm{T}$ be a theory such that $\mathrm{RIE}_{0}^{1} \subseteq \mathrm{T} \subseteq \mathrm{I} \Delta_{0}$ and $M \models T$. Assume that $\Phi(u)$ is a $\Pi_{1}$-formula defining a proper initial segment closed by multiplication in $M$. Then $M\left(\mathrm{I} \Delta_{0}\right) \models \forall x \Phi(x)$.

Proof. Assume $\Phi(u) \equiv \forall x \psi(x, u)$, with $\psi \in \Delta_{0}$, and let $N=M(\forall u \Phi(u))$. If $M\left(\mathrm{I} \Delta_{0}\right) \subseteq N$, the result holds.

Suppose that $N \subseteq M\left(\mathrm{I} \Delta_{0}\right)$. Let $M^{\prime}$ be a $\Sigma_{1}$-recursively saturated elementary extension of $M$, and $N^{\prime}=M^{\prime}(\forall u \Phi(u))$. Then $N^{\prime} \subseteq M^{\prime}\left(\mathrm{I} \Delta_{0}\right)$.

Let $\gamma(u) \equiv \forall x, y<u \psi(x, y)$. We claim that

$$
M^{\prime} \models \gamma(0) \wedge \forall u(\gamma(u) \rightarrow \gamma(u+1))
$$

If $a \in M^{\prime}$ is such that $M^{\prime} \models \gamma(a)$, then $a^{\frac{1}{\omega}} \subseteq N^{\prime}$ (because $a^{\frac{1}{\omega}} \models \forall u \Phi(u)$ ). By $\Sigma_{1}$-recursive saturation, it can to conclude that $a^{\frac{1}{\omega}} \neq N^{\prime}$. Thus, $a \in N^{\prime}$, hence $a+1 \in N^{\prime}$. Therefore, $M^{\prime} \models \gamma(a+1)$.

By induction on $\gamma(u), M^{\prime}\left(\mathrm{I} \Delta_{0}\right) \models \forall u \gamma(u)$. This implies that $N=M\left(\mathrm{I} \Delta_{0}\right)$ (because $M\left(\mathrm{I} \Delta_{0}\right) \backslash N \subseteq$ $\left.N\left(M^{\prime}, \mathrm{I} \Delta_{0}\right) \backslash N^{\prime}\right)$. 
The section will be completed by demonstrating a necessary and sufficient condition on the $\Pi_{1}$ interpretability of $\mathrm{I}_{0}$ in $\mathrm{RIE}_{0}^{1}$ which is consequence of above theorem.

Corollary 3.18. The following conditions are equivalent:

1. $\mathrm{I} \Delta_{0}$ is finitely axiomatizable modulo $\mathrm{RIE}_{0}^{1}$.

2. There exists an extension of $\mathrm{I}_{0}$ which is $\Pi_{1}$-interpretable in $\mathrm{RIE}_{0}^{1}$.

\section{Proof.}

$(1) \Longrightarrow(2):$ If $\mathrm{I} \Delta_{0} \equiv \mathrm{RIE}_{0}^{1}+\forall u \Phi(u)$, let

$$
\Phi^{\prime}(x) \equiv \Phi(x) \wedge \forall u(u<x \rightarrow \Phi(u)) \wedge \forall u<x \forall v<x \Phi(u \cdot v) .
$$

Since $M \models \mathrm{RIE}_{0}^{1}$ it must be

$$
M\left(\forall u \Phi^{\prime}(u)\right) \subseteq M(\forall u \Phi(u)) \subseteq M\left(\mathrm{I} \Delta_{0}\right)
$$

then the formula $\Phi^{\prime}(x)$ interprets an extension of $\mathrm{I} \Delta_{0}$ in $\mathrm{RIE}_{0}^{1}$.

$(2) \Longrightarrow(1)$ : Let $\Phi(x)$ the $\Pi_{1}$-interpretation of an extension $T$ of $\mathrm{I} \Delta_{0}$. If $M \models \mathrm{RIE}_{0}^{1}$, then

$$
M(\forall u \Phi(u)) \subseteq M\left(\mathrm{I} \Delta_{0}\right)
$$

and, reasoning as in Theorem 3.17, it follows that the $\mathrm{I} \Delta_{0}$-kernel is defined by $\Phi(u)$ in every model of $\mathrm{RIE}_{0}^{1}$. Therefore

$$
\mathrm{RIE}_{0}^{1}+\forall u \Phi(u) \equiv \mathrm{I} \Delta_{0}
$$

\subsection{Arithmetic of gaps and segments}

Several technical results about an extension of the model, where the gaps are considered as elements, are described in this section. A convenient way to do this is to consider them as if they were done with Henkin's cuts. This section, will introduce how arithmetic is in that extended model (see [32,33] for a study with a broader perspective of the idea). On the topology induced by cuts, in the case of PA there are studies on its behavior (see e.g. [31] where indicators are used).

Definition 3.19. Let $\varphi(x, \vec{a})$ and $\psi(x, \vec{b})$ be $L(M)$-formulas. It is said that $\varphi(x, \vec{a})$ and $\psi(x, \vec{b})$ define the same gap, $M \models \psi \sim \varphi$, if

$$
M \models \forall u \forall v(u<\varphi<v \leftrightarrow u<\psi<v)
$$

Clearly, ' $\sim$ ' is an equivalence relation. It will be denoted by $[\varphi]$ the equivalence class of $\varphi$. Whilst it is understood that the definitions in this section are intended for gaps, note that they are also suitable for $\Delta_{0}$-definable sets with a first element, by identifying each $a \in M$ with $\left[\varphi_{a}\right]$ where $\varphi_{a}(x):=a \leq x$. Thus,

$$
\text { If } M \models(\mu x) \varphi(x)=a \text { then } M \models a \sim \varphi
$$

For convenience it will be written $[a]$ or simply $a$ instead of $\left[\varphi_{a}\right]$.

Given $X \subseteq M$, it is defined

$$
\mathcal{G}_{k}(M, X):=\left\{[\varphi]: \varphi \text { is a } \mathrm{E}_{k} \text {-gap with parameters in } X\right\}
$$


and it will be written $\mathcal{G}_{k}(M)$ if $X=M$. It is possible to extend the order $<_{M}$ to $\mathcal{G}_{k}(M, X)$ as follows:

$$
M \models \varphi<\psi \text { if and only if } M \models \exists x(\varphi(x) \wedge x<\psi)
$$

The segment determined by a formula $\varphi$ is defined as

$$
S(\varphi):=\{a \in M: M \models a<\varphi\}
$$

A useful simplification is to work only with coinitial gaps. A formula $\varphi(x)$ is coinitial if $S(\varphi)$ agree with $\neg \varphi(M)$. It is obvious that every gap is equivalent to a coinitial gap.

Please note that if $X \nsubseteq S(\varphi)$, then the formula $\varphi<\psi$ is $\Delta_{0}$. It is also defined

$$
(\varphi, b):=\{x: M \models \varphi<x \wedge x<b\}
$$

Let $\varphi, \psi \in \mathcal{G}_{k}(M, X)$, with $1 \leq k \leq \omega$. The addition and the product of two gaps are defined as

$$
(\varphi * \psi)(x):=\exists u, v \leq x(\varphi(u) \wedge \psi(v) \wedge x=u * v) \quad(* \in\{+, \cdot\})
$$

Note that trivially

$$
M \models \varphi_{a} * \varphi_{b} \sim \varphi_{a * b}
$$

It is easy to see that both operations are well defined on equivalence classes, extending that of operations in $M$ as well as that they are associative and commutative $(1 \leq k \leq \omega)$. Therefore two semi-group structures are available for endowing $\mathcal{G}_{k}(M, X)$. We are interested in the idempotent gaps.

Definition 3.20. Let $* \in\{+, \cdot\}$. A gap $\varphi \in \mathcal{G}_{k}(M, X)$ is idempotent with respect to $*$ (briefly, is an $i d(*)$-gap) if $M \models \varphi * \varphi \sim \varphi$.

That is, $[\varphi]$ is an idempotent element in the semigroup $\left(\mathcal{G}_{k}(M, X), *\right)$. It will be denoted by $I_{k}^{*}(M, X)$ the set of idempotents for $*$ and parameters in $X$ (if $X=M$ it will be written $I_{k}^{*}(M)$ ).

It is easy to write a formula $\operatorname{Idemp}_{*}(\varphi)$ expressing that $\varphi$ is a $i d(*)$-gap in $\mathrm{PA}^{-}$. In the case of the theory $\mathrm{RIE}_{0}^{1}$, the following formula can be used:

$$
\operatorname{Idemp}(\varphi) \equiv \forall x, y(x<\varphi \wedge y<\varphi \rightarrow x \cdot y<\varphi)
$$

and, in $\operatorname{IE}_{0}^{1}, \operatorname{Idemp}_{+}(\varphi)$ is equivalent to a similar formula, due to the following result:

Proposition 3.21. Let $M \models P A^{-}$and let $\varphi \in \Delta_{0}$.

1. If $M \models \mathrm{IE}_{0}^{1}, \varphi$ is an id(+)-gap if and only if $S(\varphi)$ is closed by + .

2. If $M \models \mathrm{RIE}_{0}^{1}$ then $\varphi$ is an id $(\cdot)$-gap if and only if $S(\varphi) \models \mathrm{PA}^{-}$. This fact is not true for models of $\mathrm{IE}_{0}^{1}$ in general.

Proof. Proof of (2): Assume $\varphi(x)$ is idempotent and $a, b \in S(\varphi)$ such that $M \models \varphi<a \cdot b$. Then there exists $c$ such that $M \models \varphi(c) \wedge c<a \cdot b$. Let $d, e \in M$ such that

$$
M \models \varphi(d) \wedge \varphi(e) \wedge d \cdot e<c
$$

Then one of $\{d, e\}$ is less than one of $\{a, b\}$; suppose $d<a$ for example. Thus $\varphi<a$, a contradiction. 
Conversely, suppose that $\varphi(x)$ and $a \in M$ such that $M \models \varphi<a$. If $S(\varphi) \models \mathrm{PA}^{-}$, then $M \models \varphi<\lfloor\sqrt{a}\rfloor$. Therefore there exists $c \in M$ such that $M \models \varphi(c) \wedge c<\lfloor\sqrt{a}\rfloor$. Thus

$$
M \models(\varphi \cdot \varphi)\left(c^{2}\right) \wedge c^{2}<a
$$

The proof of (1) is similar, replacing · by + , and $\lfloor\sqrt{a}\rfloor$ by $\left\lfloor\frac{a}{2}\right\rfloor$.

Finally it will show that 3.21.(2) fails for a model of $\mathrm{IE}_{0}^{1}$ : Let $M_{1} \models \mathrm{IE}_{0}^{1}$ the model defined in Theorem 3.8.(2), and $\varphi(u) \equiv X \leq u^{2}$. It holds that $S(\varphi) \models \mathrm{PA}^{-}$and

$$
(\varphi \cdot \varphi)(u) \equiv \exists u_{1} u_{2}<u\left(X \leq u_{1}^{2} \wedge X \leq u_{2}^{2} \wedge u=u_{1} \cdot u_{2}\right) .
$$

Since it is verified that $M_{1} \models(\varphi \cdot \varphi)(u) \rightarrow u>X$ and $M_{1} \models \varphi(X)$, then $M_{1} \models \varphi<\varphi \cdot \varphi$.

The following result, essentially due to J. Paris [41], shows that it is possible to restrict ourselves to idempotent gaps.

Theorem 3.22. Let $M \models P A^{-}$.

1. If $M \models \mathrm{IE}_{0}^{1}, M \models \mathrm{I} \Delta_{0}$ if and only if $M$ has no bounded id(+)-gap.

2. If $M \models \mathrm{RIE}_{0}^{1}, M \models \mathrm{I} \Delta_{0}$ if and only if $M$ has no bounded id(.)-gap.

The proof of 3.22.(1) is based in the scheme

$$
\mathrm{I}_{\varphi}^{+}:=\varphi(0) \wedge \varphi(1) \wedge \forall x, y(\varphi(x) \wedge \varphi(y) \rightarrow \varphi(x+y)) \rightarrow \forall x \varphi(x)
$$

It verifies that $\mathrm{I} \Delta_{0} \equiv \mathrm{I}^{+} \Delta_{0}$ ([41], lemma 2). The fact 3.22.(2) is proved like th. 3 of [41] (using for example the set

$$
\left\{2^{a}: M \models \exists y 2^{a}=y\right\}
$$

instead of the enumeration of the roots of the equation $\left.x^{2}+y^{2}-2 a x y-1=0\right)$.

Corollary 3.23. If $M \models \mathrm{RIE}_{0}^{1}$ is $k$-high, then $M\left(\mathrm{IE}_{k}\right)$ is $\Delta_{0}$-definable.

Proof. Suppose that $a^{\frac{1}{\omega}}=M\left(\mathrm{IE}_{k}\right)$. Since $a^{\omega} \not \models \mathrm{IE}_{k}$, by 3.22 there exists $\varphi \in \mathrm{E}_{k}$ an $i d(\cdot)$-gap such that

$$
M\left(\mathrm{IE}_{k}\right)<\varphi<a^{\omega}
$$

By 3.21 it has $S(\varphi) \models \mathrm{PA}^{-}$. Therefore the only possibility is that $S(\varphi)=M\left(\mathrm{IE}_{k}\right)$. Thus the formula

$$
\psi(x) \equiv x<\varphi
$$

defines $M\left(\mathrm{IE}_{k}\right)$.

Using Corollary 3.23 it is possible to improve Theorem 3.10:

Corollary 3.24. If there exists $M \models \mathrm{RIE}_{0}^{1}$ with $M\left(\mathrm{IE}_{k}\right)$ not $\Delta_{0}$-definable, then $\mathrm{IE}_{k} \nvdash \mathrm{I} \Delta_{0}$.

Proof. If $M$ were such a model, then it would be $k$-low by the previous corollary. So, by Theorem 3.10 it follows that $\mathrm{IE}_{k} \nvdash \mathrm{I} \Delta_{0}$. 
A key property of $I_{k}^{*}(M)$ is that it is a subsemigroup itself. The result below is proved like Proposition 3.21.

Proposition 3.25. Let $[\varphi],[\psi] \in I_{k}^{*}(M)$, and $M \models \mathrm{IE}_{0}^{1}$ if $*=+$, or $M \models \mathrm{RIE}_{0}^{1}$ if $*=\cdot$. Then

$$
[\varphi] *[\psi]=\max \{[\psi],[\varphi]\} \quad * \in\{+, \cdot\}
$$

Above result contrasts with an old unsolved question on $\beta \mathbb{N}$ that clearly exposes the complexity of arithmetic in $\beta \mathbb{N}$ : Are there $p, q, r, s \in \beta \mathbb{N}^{*}$ such that $p+q=r \cdot s$ ? ([48], Chapter 7, Question 39. See also [1] for other similar problems).

Both operations present similar properties under the proper base theory ( $\mathrm{IE}_{1}$ or $\mathrm{RIE}_{0}^{1}$ ), so only the results for the product will be demonstrated. We only enunciate the corresponding ones for the addition.

Consider the structure $\left(M\left[\mathcal{G}_{k}\right],+, \cdot, \leq\right)$ whose universe is the set of equivalence classes of $E_{k}$-definable sets (identifying the element $a$ with $\varphi_{a}$ ), and the symbols are interpreted as before. Note that $M\left[\mathcal{G}_{k}\right] \not \forall \mathrm{PA}^{-}$ in general, because $M\left[\mathcal{G}_{k}\right] \models \exists x(x=x+1)$ when $M \not \models \mathrm{IE}_{k}$ (by Proposition 3.25). In fact, the relationship between $M\left[\mathcal{G}_{k}\right]$ and $M$ can be determined by relativizing the formulas to $M$ via the natural map $\varphi \mapsto \varphi^{\prime}$ that relativizes to elements $x$ such that $x \neq x+1$.

Since $\mathcal{G}_{k}(M)$ is defined in $M\left[\mathcal{G}_{k}\right]$ by the formula ' $x=x+1$ ', then for every $L(M)$-sentence $\varphi$,

$$
M \models \varphi \text { if and only if } M\left[\mathcal{G}_{k}\right] \models \varphi^{\prime}
$$

On the other hand, since $\mathbb{N} \subset_{e} M\left[\mathcal{G}_{k}\right]$, it is verified that $M\left[\mathcal{G}_{k}\right] \models \Sigma_{1}(\mathbb{N})$ (the true $\Sigma_{1}$-sentences in $\mathbb{N}$ ). In general, in $\mathrm{IE}_{k}$-models, if $T h\left(M\left[\mathcal{G}_{k}\right]\right) \vdash x \neq x+1$ then $M \models \mathrm{IE}_{k+1}$.

\section{Part II: algebraic combinatorics in $\mathrm{IE}_{k}$}

The results of the first part will now allow us to both enunciate and prove properties in weak induction models that relate combinatorial properties and sets without a first element (gaps). The task will then be carried out concerning the existence of relative composite numbers in some sets, following the idea described in the introduction.

\subsection{Combinatorics in gaps}

The previous section has provided two semigroup structures on $M\left[\mathcal{G}_{k}\right]$. Therefore, one is in a position to apply the (combinatorial) results on topological semigroups to the new structures, to later describe the results in terms of the starting model $M$.

The next aim is to state several combinatorial properties about the upper neighborhood of an idempotent gap (that is, intervals like $(\varphi, a))$ in the semigroup $\left(M\left[\mathcal{G}_{k}\right], *\right)(* \in\{+, \cdot\})$, formalizing the idea about prime numbers relative to a set that was described in the introduction.

For the study it is convenient to split the interval $(\varphi, \infty)$ into $\varphi(M)$ and its complement within the same interval:

Definition 4.1. Let $\varphi(x)$ be an $\mathrm{E}_{k}$-gap. The complement of $\varphi$ is the formula $\bar{\varphi}(x):=\neg \varphi(x) \wedge \varphi<x$.

The definition of composite number related to a set (relative composite number) would be defined as follows:

Definition 4.2. Let $\varphi \in \mathrm{E}_{k}$ and $M \models \mathrm{PA}^{-}$. An element $a \in M$ is $\varphi$-composite with respect to $* \in\{+, \cdot\}$ if $M \models \varphi_{\text {comp* }}(a)$, where

$$
\varphi_{\text {comp* }}(x):=\varphi(x) \wedge \exists u_{1}, u_{2} \leq x\left(x=u_{1} * u_{2} \wedge \varphi\left(u_{1}\right) \wedge \varphi\left(u_{2}\right)\right)
$$


That is

$$
\varphi_{c o m p *}(M)=(\varphi(M) * \varphi(M)) \cap \varphi(M)
$$

The translation of arithmetical properties (e.g. prime, irreducible elements) to Stone-Čech compactification is not direct and it raises very interesting questions [45]. Please observe that, in general,

$$
M \not \models \varphi_{\text {comp* }} \sim \varphi * \varphi
$$

If $0,1 \notin \varphi(M)$ and $\varphi(M)$ has a first element, then the set $\varphi_{\text {comp* }}(M)$ is not coinitial with $\varphi($ nor $\bar{\varphi})$ composite elements, because the first element of $\varphi(M)$ does not satisfy $\varphi_{\text {comp* }}$, that is, is a prime relative. Analogously with $\bar{\varphi}_{\text {comp* }}$. Therefore, the following translation of the arithmetical property $(\diamond)$ discussed in the introduction is satisfied.

Proposition 4.3. Assume $M \models \mathrm{IE}_{0}^{1}$ and $\varphi$ is an $L(M)$-formula. Then

$$
M \models \neg \varphi(0) \wedge \neg \varphi(1) \wedge \exists x \varphi(x) \rightarrow\left(\mathrm{L}_{\varphi} \rightarrow \varphi_{c o m p *}>\varphi \wedge(\bar{\varphi})_{c o m p *}>\varphi\right) \quad(* *)
$$

Proof. Trivial.

Please observe that for proving the reciprocal of Proposition 4.3 (namely, if $M$ verifies $A \nsim_{S} A^{*}$ for any nontrivial $\Delta_{0}$-definable set $A$, then is a model of $\mathrm{I} \Delta_{0}$ ) can not be straightforwardly proved. The existence of relative composite elements arbitrarily close to the gap needs to be demonstrated (actually it is just needed to state this feature for idempotent $\Delta_{0}$-gaps). The property can be demonstrated by showing that $\varphi(M)$ satisfies some Ramsey type regularity for a certain kind of gap $\varphi$.

\subsection{FP and FS-sets within gaps}

The regularity feature required to prove the reciprocal of Proposition 4.3 is stated in Theorem 4.4, which is a version of the finite sums theorem (Theorem 4.4). Its proof is inspired by that of Galvin-Glatzer's proof of the classic result on FP-sets, namely by exploiting the algebraic structure of $\beta M$. Specifically, it is shown that given $\varphi$ a gap, this or its complement contains FP-sets arbitrarily close to $[\varphi]$.

Theorem 4.4. Let $M \models \mathrm{IE}_{0}^{1}+\neg \mathrm{IE}_{k}$, and let $\varphi$ be an idempotent $E_{k}$-gap with respect to $\cdot$ Then there exists $\psi \in\{\varphi, \bar{\varphi}\}$ such that $\psi(M) \cap(\psi, c)$ is a FP-set for arbitrarily $c>\varphi$.

Proof. Consider the closed (hence compact) nonempty set in $\beta M$ (recall that, in the Stone-Čech compactification, $\bar{A}$ denotes the closure of the set $A \subseteq M$ in $\beta M$ )

$$
\mathbb{H}_{\varphi}:=\bigcap_{\varphi<a} \overline{\{b: M \models \varphi<b \wedge b<a\}}
$$

We claim that

$\underline{\text { Claim }} \mathbb{H}_{\varphi}$ is a subsemigroup of $\beta M$.

Proof of the Claim: It is necessary to prove that $p \cdot q \in \mathbb{H}_{\varphi}$ for any $p, q \in \mathbb{H}_{\varphi}$. That is, that $(\varphi, a) \in p \cdot q$, for all $a>\varphi$; or equivalently,

$$
\{x:\{y: x \cdot y \in(\varphi, a)\} \in q\} \in p
$$

Write $x^{-1}(\varphi, a)=\{y: x \cdot y \in(\varphi, a)\}$. Let us observe that $x^{-1}(\varphi, a) \in q$ if and only if $\varphi<\left\lfloor\frac{a}{x}\right\rfloor$ : 
If $\varphi<\left\lfloor\frac{a}{x}\right\rfloor$, then $\left(\varphi,\left\lfloor\frac{a}{x}\right\rfloor\right) \in q$ hence $x^{-1}(\varphi, a) \in q$. Otherwise $\left\lfloor\frac{a}{x}\right\rfloor<\varphi$, then

$$
(\varphi, a) \cap x^{-1}(\varphi, a)=\emptyset
$$

hence $x^{-1}(\varphi, a) \notin q$.

Therefore, it is sufficient to show

$$
\left\{x: \varphi<\left\lfloor\frac{a}{x}\right\rfloor\right\} \in p
$$

Since the set is convex and $p \in \mathbb{H}_{\varphi}$, the condition above is equivalent to the existence of $x>\varphi$ such that $\varphi<\left\lfloor\frac{a}{x}\right\rfloor$. Let us see that such an element exists:

Since $M \models \varphi \sim \varphi \cdot \varphi$, there is $d<a$ such that

$$
M \models(\varphi \cdot \varphi)(d) \wedge d<a
$$

so $M \models \varphi\left(d_{1}\right) \wedge \varphi\left(d_{2}\right) \wedge d_{1} \cdot d_{2}=d$ for some $d_{1}, d_{2}$. If $x=d_{1}$, then $\left\lfloor\frac{a}{x}\right\rfloor>\left\lfloor\frac{d}{x}\right\rfloor=d_{2}$ and $M \models d_{2}>\varphi$, because $M \models \varphi\left(d_{2}\right)$.

end of claim proof

According to Result 2.4, it is known that $\mathbb{H}_{\varphi}$ contains an idempotent element $p$. Given $c>\varphi$, since

$$
\{(\varphi, c) \cap \varphi(M),(\varphi, c) \cap \bar{\varphi}(M)\}
$$

is a partition of $(\varphi, c) \in p$, there is $\psi \in\{\varphi, \bar{\varphi}\}$ such that

$$
\psi(M) \cap(\psi, c) \in p
$$

hence this set is a $F P$-set.

It only remains to prove that the choice of $\psi$ is valid for any element $c^{\prime}>\varphi$, (that we can suppose $c^{\prime}<c$ ). That fact is true, because necessarily $\left(\varphi, c^{\prime}\right) \cap \psi(M) \in p$ (otherwise we would have $\left(\varphi, c^{\prime}\right) \cap \bar{\psi}(M) \in p$, but this set would disjoint with $\psi(M) \cap(\varphi, c)$, that is not possible).

Please observe that it is easy to see that $\pi: \beta M \rightarrow M[\mathcal{G}]$ of Fig. 1 verifies $\pi\left[\mathbb{H}_{\varphi}\right]=\{[\varphi]\}$, as well as the converse of the above result: If $\varphi(M)$ contains FP-sets arbitrarily near the gap, then it is an $i d(\cdot)$-gap. Without any essential modification, a similar result would be obtained for $i d(+)$-gaps can be proved:

Theorem 4.5. Let $M \models \mathrm{IE}_{0}^{1}+\neg \mathrm{IE}_{k}$, and let $\varphi(x) \in \mathrm{E}_{k}$ be an id(+)-gap. There is $\psi \in\{\varphi, \bar{\varphi}\}$ such that $\psi(M)$ contains a FS-set arbitrarily near the gap; that is, for any $c>\varphi, \psi(M) \cap(\psi, c)$ is a FP-set.

A consequence of the above theorems was remarked above (directly provable by Theorem 4.4, by using Theorem 3.22):

Corollary 4.6. Suppose that $\varphi$ be a $\Delta_{0}$-formula in the language $L(M)$ such that

$$
M \models \operatorname{Idemp}_{*}(\varphi) \wedge \neg \varphi(0) \wedge \neg \varphi(1)
$$

and $M \models \mathrm{IE}_{0}^{1}$ if $*=+$ or $M \models \mathrm{RIE}_{0}^{1}$ if $*=$. If $M \models \neg \mathrm{L}_{\varphi}$, then

$$
M \models \varphi_{c o m p *} \sim \varphi \vee(\bar{\varphi})_{c o m p *} \sim \varphi
$$




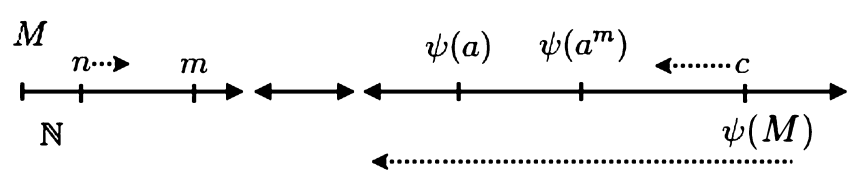

Fig. 3. Existence of arbitrary large powers in gaps (Theorem 4.10).

Therefore, by instantiating the result into a scheme that reflects that property alternative axiomatizations of I $\Delta_{0}$ are available. The following corollary shows an axiomatic characterization of I $\Delta_{0}$ using only the arithmetic properties that have been introduced: an idempotent set that is coinitial with its relative composite numbers contains either 0 or 1 .

Corollary 4.7. The following theories are equivalent to $\mathrm{I} \Delta_{0}$ :

$$
\begin{aligned}
& \operatorname{RIE}_{0}^{1}+\left\{\exists x \varphi(x) \wedge \operatorname{Idemp}(\varphi) \wedge\left(\varphi_{\text {comp }} \sim \varphi \vee(\bar{\varphi})_{c o m p .} \sim \varphi\right) \rightarrow(\varphi(0) \vee \varphi(1))\right\}_{\varphi \in \Delta_{0}} \\
& \operatorname{IE}_{0}^{1}+\left\{\exists x \varphi(x) \wedge I \operatorname{Iemp}_{+}(\varphi) \wedge\left(\varphi_{\text {comp }} \sim \varphi \vee(\bar{\varphi})_{c o m p+} \sim \varphi\right) \rightarrow(\varphi(0) \vee \varphi(1))\right\}_{\varphi \in \Delta_{0}}
\end{aligned}
$$

Exploiting the ideas introduced in this subsection, richer combinatorial properties can be proved if we allow the model to fulfill basic additional properties, such as overspill for its standard part.

\subsection{Combinatorics in presence of weak overspill on $\mathbb{N}$}

This section will show some results from semi-group properties $\mathbb{H}_{\varphi}$ (introduced in Theorem 4.4) for models which satisfies a weak form of overspill (see [15]):

Definition 4.8. Let $M \models \mathrm{PA}^{-}$and $\Gamma$ a class of formulas. It is said that $M$ has overspill $\Gamma$ on $\mathbb{N}, M \models$ $\mathbb{N}-O(\Gamma)$, if for all $\varphi(x, \vec{u}) \in \Gamma, \vec{c} \in M$ and $\beta>\omega$, if

$$
\forall n \in \omega \exists m>n[M \models \varphi(m, \vec{c})]
$$

then there is $\delta<\beta$ nonstandard such that $M \models \varphi(\delta, \vec{c})$.

This is a weak notion of overspill; any $\Delta_{0}$-recursively saturated model of $\mathrm{PA}^{-}$has $\mathbb{N}-O\left(\Delta_{0}\right)$. Nevertheless, sometimes it is a good substitute for $\Delta_{0}$-recursive saturation; for example 3.8.(2) is true for models of $\mathrm{IE}_{k_{e}}+\mathbb{N}-O\left(\Delta_{0}\right)$, thus several results of Fig. 2 could be refined for these kinds of models.

Theorem 4.10 will show that, in models satisfying $\mathbb{N}-O(\Gamma)$, the regularity property can be refined by replacing the FP-sets with (standard) powers of an element. This will allow another alternative axiom of $\mathrm{I} \Delta_{0}$ using the formula $\exp$ (Corollary 4.14).

Definition 4.9. A set $A \subseteq M \models P A^{-}$is said to be dense for standard powers if

$$
\forall c \forall n \in \omega\left[[0, c] \cap A \neq \emptyset \quad \Longrightarrow \quad \exists a<c \exists m>n\left(a, a^{m} \in A\right)\right]
$$

In the following theorem the density for standard powers for gaps (Fig. 3) is stated:

Theorem 4.10. Let $M \models \mathrm{RIE}_{0}^{1}+\neg \mathrm{IE}_{k}$, and $\varphi(x) \in E_{k}$ be an id(·)-gap. Then there exists $\psi \in\{\varphi, \bar{\varphi}\}$ such that $\psi(M)$ is dense in standard powers.

Proof. The function $f(x):=x^{2}$ has a continuous extension $\beta f: \beta M \rightarrow \beta M$. That function has not any fixed point in $\beta M \backslash\{0,1\}$ by Result 2.6. 
Since $f[(\varphi,\lfloor\sqrt{b}\rfloor)] \subseteq(\varphi, b)$, it follows that $(\varphi, b) \in \beta f(p)$, for all $p \in \mathbb{H}_{\varphi}$ and $b>\varphi$, so it defines the restriction

$$
\beta f \uparrow_{\mathbb{H}_{\varphi}}: \mathbb{H}_{\varphi} \rightarrow \mathbb{H}_{\varphi}
$$

Therefore it can be considered the dynamic system:

$$
\left(\mathbb{H}_{\varphi},\left\{(\beta f)^{n}\right\}_{n \in \omega^{+}}\right)
$$

on the compact space $\mathbb{H}_{\varphi}\left(\omega^{+}=\omega \backslash\{0\}\right)$.

By Result 2.7 there exist $p \in \mathbb{H}_{\varphi}$ and $r \in \beta\left(\omega^{+}\right)$such that $(\beta f)^{r}(p)=p$.

That means that for all $A \in p$ that is, for all $\bar{A}$ neighborhood of $p$

$$
\left\{n \in \omega^{+}:(\beta f)^{n}(p) \in \bar{A}\right\} \in r .
$$

Since $(\beta f)^{n}=\beta\left(f^{n}\right)$ and $f^{n}[A] \in \beta\left(f^{n}\right)(p)$, it follows that

$$
\left\{n \in \omega^{+}: f^{n}[A] \cap A \neq \emptyset\right\} \in r .
$$

Since $r$ is not principal (because $0,1 \notin \mathbb{H}_{\varphi}$ ),

$$
\forall n \exists m>n\left[f^{m}[A] \cap A \neq \emptyset\right]
$$

Let $\psi \in\{\varphi, \bar{\varphi}\}$ such that the set $A=\psi(M) \cap(\varphi, c)$ belongs in $p$. Then, by ( $\dagger)$,

$$
\forall n \exists m>n \text { such that } M \models \exists x<c\left[\psi(x) \wedge \psi\left(x^{2^{m}}\right)\right]
$$

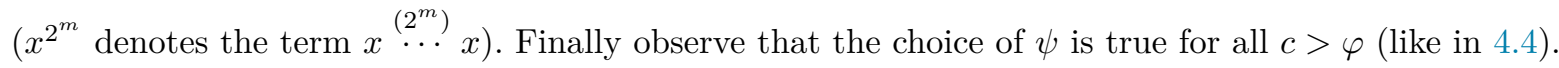

We will use again the index $k_{e}$ (see 2.3) to show, in models of $\mathrm{IE}_{k_{e}}+\mathbb{N}-O\left(\mathrm{E}_{k}\right)$, a nonlinear relation near the gap.

Definition 4.11. A set $A \subseteq M \models P A^{-}$is said to be dense for powers if

$$
\forall c\left[[0, c] \cap A \neq \emptyset \quad \Longrightarrow \quad \exists a \in A \exists \delta>\omega\left(a, a^{\delta} \in A\right)\right]
$$

Corollary 4.12. Under the conditions of Theorem 4.10, if $M \models \mathrm{IE}_{k_{e}}+\mathbb{N}-O\left(\mathrm{E}_{k}\right)$, then there is $\psi \in\{\varphi, \bar{\varphi}\}$ such that $\psi(M)$ is dense for powers.

Proof. Let $k>k_{e}$ Let $x^{y}=z$ be the formula defined in Sect. 2. It has that, for all $n \in \omega$,

$$
\mathrm{IE}_{k_{e}} \vdash x \stackrel{(n)}{\cdots} x=y \rightarrow x^{n}=y
$$

By 4.10, we have that for all $n \in \omega$ there is $m>n$ such that

$$
M \models \exists x, y<c\left(x^{m}=y \wedge \psi(x) \wedge \psi(y)\right)
$$

Since the formula is $\mathrm{E}_{k}\left(1 \leq k_{e} \leq k\right)$, by applying $\mathbb{N}-O\left(\mathrm{E}_{k}\right)$, the result is concluded.

Corollary 4.13. If $M \models \mathrm{IE}_{k_{e}}+\neg \mathrm{IE}_{k}+\mathbb{N}-O\left(\mathrm{E}_{k}\right)$, then $M$ is $k$-low. 
Proof. Suppose $M\left(\mathrm{IE}_{k}\right) \subseteq a^{\frac{1}{\omega}}$. Let $\varphi \in \mathrm{E}_{k}$ be a $i d(\cdot)$-gap such that

$$
M\left(\mathrm{IE}_{k}\right)<\varphi<a
$$

Reasoning like 4.12 , there is $\psi \in\{\varphi, \bar{\varphi}\}$ such that

$$
M \models \exists x<a\left(x^{2^{m}}<a \wedge \psi(x) \wedge \psi\left(x^{2^{m}}\right)\right)
$$

By $\mathbb{N}-O\left(\mathrm{E}_{k}\right)$, there is $b \in M$ and $\delta>\omega$ such that $M \models \psi(b) \wedge \psi\left(b^{\delta}\right) \wedge b^{\delta}<a$. Thus, $M\left(\mathrm{IE}_{k}\right)<b<a^{\frac{1}{\omega}}$.

The above results allow obtaining other alternative axiomatizations of $\mathrm{I} \Delta_{0}$. Following the idea of the relative composite numbers, given $\varphi(x)$ it is defined the exponential relative to $\varphi$ as the formula

$$
\varphi_{\text {exp }}(x):=\varphi(x) \wedge \exists y, z<x\left[z \neq 1 \wedge y^{z}=x \wedge \varphi(y)\right]
$$

Corollary 4.14. $\mathrm{I} \Delta_{0}$ is equivalent to

$$
\operatorname{IE}_{k_{e}}+\left\{\operatorname{Idemp} \bullet(\varphi) \wedge \neg \varphi(0) \wedge \neg \varphi(1) \wedge \exists x \varphi(x) \rightarrow \varphi<\varphi_{\exp } \wedge \varphi<(\bar{\varphi})_{e x p}\right\}_{\varphi \in \Delta_{0}}
$$

If it is replaced - by + and the map $x \mapsto 2 x$ is used instead of $x \mapsto x^{2}$, then -by a similar argument of above proofs- an axiomatization similar to that of Corollary 4.14 can be obtained, because it has the following result:

Theorem 4.15. Let $M \models \mathrm{IE}_{0}^{1}+\neg \mathrm{IE}_{k}$, and $\varphi(x) \in \Delta_{0}$ be an $i d(+)$-gap. Then there exists $\psi \in\{\varphi, \bar{\varphi}\}$ such that for all $c>\varphi$ and $n \in \omega$, there exist $m>n$ and $a \in(\varphi, c)$ such that

$$
M \models \psi(a) \wedge \psi\left(2^{m} \cdot a\right)
$$

Moreover, if $M \models \mathrm{IE}_{k_{e}}+\mathbb{N}-O\left(\mathrm{E}_{k}\right)$, then there exists $\psi \in\{\varphi, \bar{\varphi}\}$ such that for all $c>\psi$ there exists $a \in(\varphi, c)$ and $\delta$ nonstandard such that

$$
M \models \psi(a) \wedge \psi\left(2^{\delta} \cdot a\right)
$$

\section{Closing remarks}

The starting thesis of the article was to advance in the study of models of very weak arithmetic theories by considering different methods from the classics that need more resources (those based on recursiontheoretic methods, definability of satisfaction, etc.). On the one hand, it has been proven that we can reduce some problems to the existence of a certain model or of some $\Delta_{0}$-definable set. Axiom schemes describing properties of algebro-combinatoric nature for weak induction have also been provided, thus complementing related results for other theories without $\Sigma_{1}$ induction (see e.g. $[18,52]$ ).

In the paper it has been shown that the use of ultrafilters of the set $\mathbb{S}_{\varphi}=\pi^{-1}[\{[\varphi]\}]$ seems to be a good substitute to the use of types in recursive saturation: it proves the existence of an element (e.g. a $\varphi$-composite element) without realizing $\Delta_{0}$-types. However, is not used the full ultrafilter, actually we work with $p \cap \nabla_{k}(M)$ instead of $p \in \beta M$ (where $\nabla_{k}(M)$ is the class of both $E_{k}$ and $U_{k}$ definable sets in $M$ ), which may be seen as a $\nabla_{k}$-type.

Nonetheless, it should be pointed out that the restriction to $\Delta_{0}$-definability limits the algebraic methods designed for $\beta \mathbb{N}$. A closer look reveals that the product has a complex behavior on the set of idempotents of $\mathbb{H}_{\varphi}$ : 
Proposition 5.1. If $M \models R \mathrm{IE}_{0}^{1}$ is countable and $\psi<\varphi$ are gaps, then the left translation defined by an idempotent $q \in \mathbb{S}_{\psi}$ is not trivial on $\mathbb{S}_{\varphi}$ (although we know that $[\psi] \cdot[\varphi]=[\varphi]$ for gaps): there is $p \in \mathbb{S}_{\varphi}$ idempotent such that $q \cdot p \neq p$ for all idempotent $q \in \bigcup_{\psi<\varphi} \mathbb{S}_{\psi}$.

Proof. Let us consider the semigroup $M_{1}=M \backslash\{0\}$. Since $\mathbb{S}_{\varphi}$ is a $G_{\delta}$-set in $\beta M_{1}$ that contains idempotents, we have that there exists $p \in \mathbb{S}_{\varphi}$ such that for every idempotent $q \in \beta M_{1} \backslash M_{1}$,

$$
q \cdot p=p \quad \Longrightarrow \quad p \cdot q=q
$$

([24], th. 9.7). If $q \in \bigcup_{\psi<\varphi} \mathbb{S}_{\psi}$, then $\pi(p \cdot q)=\pi(p) \cdot \pi(q)=[\varphi] \cdot[\psi]=[\varphi]$ (by 3.25). Thus $q \cdot p \neq p$.

Result 5.1 is also true for $\mathbb{H}_{\varphi}$, and it suggests the hardness of defining a semigroup embedding

$$
i: M\left[\mathcal{G}_{k}\right] \hookrightarrow \beta M
$$

such that $i \uparrow_{M}=I d_{M}$ with interesting features. Results like above illustrate that the algebraic combinatorics of $\beta M$ have interest beyond the topic of the paper (Weak Arithmetics). For example, the availability of such an embedding can be useful for the end-extension problem for $\mathrm{I} \Delta_{0}$. Indeed we hope to follow this research line.

Nevertheless, we also remark that it is possible to find an algebraic and topological representation of $\beta M$ (being $M$ countable) in terms of $\beta \mathbb{N}$ : for each $* \in\{+, \cdot\}$ there exists a surjective continuous homomorphism

$$
\Phi_{*}:\left(\bigcap_{n>0}{\overline{2^{n}}}^{\beta \mathbb{N}},+\right) \rightarrow(\beta M, *)
$$

(cf. th. 6.4 of [24]). Therefore, if $\sim_{\Phi_{*}}$ is the congruence induced by $\Phi_{*}$, then

$$
\bigcap_{n>0}{\overline{2^{n}}}^{\beta \mathbb{N}} / \sim_{\Phi_{*}} \cong \beta M
$$

Similar results in that line were already available (see for example [34,35]) from seminal works by Skolem and Tennenbaum, in which they relate the nature of certain ultrafilters used to build the quotient (in our case, the construction would be by means of congruences instead). The fact motivates as future work to study the congruences in $\beta M$ that produce models of a certain arithmetic theory, particularly:

Open problem: To characterize the congruences on

$$
\bigcap_{n>0}{\overline{2^{n}}}^{\beta \mathbb{N}}
$$

that induce models of $\mathrm{I} \Delta_{0}$.

Results on that line already exist. For example, in [53] Bohr compactification of a group is characterized by a congruence that makes a certain class of ultrafilters equivalent to the identity element. This result applies to the group of integers $\mathbb{Z}_{M}$ induced by $M \models P A^{-}$.

Finally, an interesting question arises by considering the idea behind Corollary 4.12. The combinatorial apparatus built to prove Theorem 4.10 could be applied to other functions with similar behavior, for instance to those defined in the given theory by $\exists \mathrm{E}_{k}$-formulas without parameters such that $M \models x<f(x)$. It seems that 4.10 could be generalized when the formula

$$
f(\varphi)(x):=\exists u<x(f(u)=x \wedge \varphi(u))
$$


defines the same gap as $\varphi$. From there one could try to obtain results like 4.12 , using some $\Delta_{0}$-definition of the iteration of $f$ (if this is possible), mainly for gaps $\varphi$ such that $f(\varphi) \sim \varphi$. There are reasons to expect that, in many cases, one might obtain axiom schemes between $\mathrm{IE}_{n}$ and $\mathrm{IE}_{n+1}$, suggesting the future study of the class of recursive functions with axiomatizable combinatorics (like Corollaries 4.6 or 4.14) in a theory weaker than $\mathrm{I} \Delta_{0}$.

\section{Acknowledgements}

This work is partially supported by project PID2019-109152GB-I00 funded by the Spanish Ministry of Science, Innovation and Universities (Ministerio de Ciencia, Innovación y Universidades) co-financed by FEDER funds.

I acknowledge the reviewers for their work and suggestions that have improved the paper. Also I am indebted to the anonymous reviewers of old versions of some of the results that appear in the article.

This work has been done in loving memory and in homage to my Ph.D. thesis supervisor, Prof. Alejandro Fernández-Margarit, who left us too soon. Without the formation I received from him, this paper would have not been possible.

\section{References}

[1] V. Bergelson, B. Rothschild, A selection of open problems, Topol. Appl. 156 (16) (2009) 2674-2681.

[2] A. Blass, Ultrafilters: where topological dynamics = algebra = combinatorics, Topol. Proc. 18 (1993) 33-56.

[3] J. Borrego-Díaz, A. Fernández-Margarit, M. Pérez-Jiménez, On overspill principles and axiom schemes for bounded formulas, Math. Log. Q. 42 (1) (1996) 341-348.

[4] J. Borrego-Díaz, A. Fernández-Margarit, M. Pérez-Jiménez, Bounded Induction and Feasible Computable Functions, preprint n. 39 of Faculty of Mathematics, University of Sevilla, 1997.

[5] S. Boughattas, L'arithmetique ouverte et ses modeles non-standards, J. Symb. Log. 56 (2) (1991) $700-714$.

[6] M. Carl, P. D'Aquino, S. Kuhlmann, Value groups of real closed fields and fragments of Peano arithmetic, Forum Math. 29 (4) (2012).

[7] P. Clote, J. Krajicek, Open problems, in: P. Clote, J. Krajicek (Eds.), Arithmetic, Proof Theory and Computational Complexity, Clarendon Press, 1993, pp. 1-19, Ch. 1.

[8] C. Cornaros, Weak forms of the regularity principle in the presence of ie 1 , Math. Log. Q. 59 (1-2) (2013) 84-100.

[9] P. D'Aquino, A sharpened version of McAloon's theorem on initial segments of models of i $\delta_{0}$, Ann. Pure Appl. Log. $61(1-2)(1993)$ 49-62.

[10] P. D'Aquino, Pell equations and exponentiation in fragments of arithmetic, Ann. Pure Appl. Log. 77 (1) (1996) 1-34.

[11] P. D'Aquino, J.F. Knight, S. Starchenko, Real closed fields and models of Peano arithmetic, J. Symb. Log. 75 (1) (2010) 1-11.

[12] M. Davis, Applied Nonstandard Analysis, John Wiley, New York, 1977.

[13] M. Di Nasso, I. Goldbring, M. Lupini, Nonstandard Methods in Ramsey Theory and Combinatorial Number Theory, Lecture Notes in Mathematics, Springer International Publishing, 2019, https://books.google.es/books?id=HwB wwEACAAJ.

[14] C. Dimitracopoulos, Matijasevic's theorem and fragments of arithmetic, Ph.D. thesis, University of Manchester, 1980.

[15] C. Dimitracopoulos, Overspill and fragments of arithmetic, Arch. Math. Log. 28 (3) (1989) $173-179$.

[16] C. Dimitracopoulos, On end extensions of models of subsystems of Peano arithmetic, Theor. Comput. Sci. 257 (1) (2001) 79-84.

[17] R. Ellis, Lectures on Topological Dynamics, Mathematics Lecture Note Series, W. A. Benjamin, 1969.

[18] A. Enayat, T.L. Wong, Unifying the model theory of first-order and second-order arithmetic via $W K L_{0}^{*}$, Ann. Pure Appl. Log. 168 (6) (2017) 1247-1283.

[19] J.M. Fraser, Almost arithmetic progressions in the primes and other large sets, Am. Math. Mon. 126 (6) (2019) 553-558, https://doi.org/10.1080/00029890.2019.1586264.

[20] H. Gaifman, C. Dimitracopoulos, Fragments of Peano's arithmetic and the MRDP theorem, in: Logic and Algorithmic, in: Monograph. Enseign. Math., vol. 30, Université de Genève, 1982, pp. 187-206.

[21] J. Glivická, P. Glivický, Shepherdson's theorems for fragments of open induction, arXiv:1701.02001, 2017.

[22] P. Hájek, P. Pudlák, Metamathematics of First-Order Arithmetic, Perspectives in Mathematical Logic, Springer-Verlag, 1993.

[23] S. Hetzl, T.L. Wong, Some observations on the logical foundations of inductive theorem proving, Log. Methods Comput. Sci. 13 (4) (2017).

[24] N. Hindman, D. Strauss, Algebra in the Stone-Čech Compactification: Theory and Applications, 2nd ed., De Gruyter Studies in Mathematics, Walter de Gruyter, 1998.

[25] N. Hindman, A. Maleki, D. Strauss, Linear equations in the Stone-Čech compactification of $\mathbb{m}$, Integers 0 (2000) 1-20.

[26] J. Hirshfeld, Nonstandard combinatorics, Stud. Log. 47 (3) (1988) 221-232. 
[27] E. Jeřábek, Division by zero, Arch. Math. Log. 55 (7) (2016) 997-1013.

[28] R. Kaye, Parameter-free universal induction, Math. Log. Q. 35 (5) (1989) 443-456.

[29] R. Kaye, Diophantine induction, Ann. Pure Appl. Log. 46 (1) (1990) 1-40.

[30] R. Kaye, Models of Peano Arithmetic, Oxford Logic Guides, Clarendon Press, 1991.

[31] R. Kaye, Generic cuts in models of arithmetic, Math. Log. Q. 54 (2) (2008) 129-144.

[32] R. Kaye, The arithmetic of cuts in models of arithmetic, Math. Log. Q. 59 (4-5) (2013) 332-351.

[33] R. Kaye, T.L. Wong, The model theory of generic cuts, in: Logic Without Borders - Essays on Set Theory, Model Theory, Philosophical Logic and Philosophy of Mathematics, Walter de Gruyter, 2015, pp. 281-296.

[34] J. Kennedy, On embedding models of arithmetic into reduced powers, Mat. Contemp. 24 (1) (2003) 91-115.

[35] J. Kennedy, S. Shelah, On embedding models of arithmetic of cardinality $\aleph_{1}$ into reduced powers, Fundam. Math. 176 (1) (2003) 17-24.

[36] A.P. Kreuzer, K. Yokoyama, On principles between $\Sigma 1$ - and $\Sigma$ 2-induction, and monotone enumerations, J. Math. Log. 16 (1) (2016)

[37] H. Lessan, Models of arithmetic, Ph.D. thesis, University of Manchester, 1978.

[38] K. McAloon, Studies in complexity theory, in: R. Book (Ed.), Studies in Complexity Theory, Pitman and John Wiley, 1986, pp. 119-221.

[39] V. Pambuccian, The Erdős-Selfridge and the Schinzel-Tijdeman theorems hold in pa ${ }^{-}$, arXiv:1410.8441, 2014.

[40] J. Paris, L. Kirby, $\Sigma_{n}$-collection schemas in arithmetic, in: A. Macintyre, L. Pacholski, J. Paris (Eds.), Logic Colloquium '77, in: Studies in Logic and the Foundations of Mathematics, vol. 96, Elsevier, 1978, pp. 199-209.

[41] J.B. Paris, Note on an induction axiom, J. Symb. Log. 43 (1) (1978) 113-117.

[42] J.B. Paris, On the structure of models of bounded $e_{1}$-induction, Čas. Pěst. Mat. 109 (4) (1984) $113-117$.

[43] J.C. Shepherdson, A non-standard model for a free variable fragment of number theory, Bull. Acad. Pol. Sci. XII (1964) 79-86.

[44] S.T. Smith, Quadratic residues and $x^{3}+y^{3}=z^{3}$ in models of ie 1 and ie 2 , Notre Dame J. Form. Log. 34 (3) (1993) $420-438$.

[45] B. Šobot, Divisibility in the Stone-Čech compactification, Rep. Math. Log. 50 (2015) 53-66.

[46] Y.-i. Tanaka, A. Tsuboi, A construction of real closed fields, Math. Log. Q. 61 (3) (2015) 159-168.

[47] T. Tao, B. Green, The primes contain arbitrarily long arithmetic progressions, Ann. Math. 167 (2008) $481-547$.

[48] J. van Mill, G. Reed, Open Problems in Topology, North-Holland, 1990.

[49] A. Visser, Peano corto and Peano basso: a study of local induction in the context of weak theories, Math. Log. Q. 60 (1-2) (2014) 92-117.

[50] A. Wilkie, J. Paris, On the scheme of induction for bounded arithmetic formulas, Ann. Pure Appl. Log. 35 (1987) 261-302, https://doi.org/10.1016/0168-0072(87)90066-2, http://www.sciencedirect.com/science/article/pii/0168007287900662.

[51] G. Wilmers, Bounded existential induction, J. Symb. Log. 50 (1) (1985) 72-90.

[52] K. Yokoyama, On the strength of Ramsey's theorem without $\Sigma_{1}$ induction, Math. Log. Q. 59 (1-2) (2013) $108-111$.

[53] P. Zlatoš, The Bohr compactification of an Abelian group as a quotient of its Stone-Cech compactification, arXiv:1808. 04637, 2018. 